\title{
Serum microRNAs as potential new biomarkers for cisplatin resistance in gastric cancer patients
}

\author{
Lei Jin Equal first author, 1, 2, Nan Zhang ${ }^{\text {Equal first author, 2, } 3 \text {, Qian Zhang }}{ }^{2,4}$, Guoqian Ding ${ }^{\text {1, }}{ }^{\text {, }}$ Zhenghan Yang ${ }^{\text {Corresp., 2, } 3}$, Zhongtao \\ Zhang ${ }^{\text {Corresp. 1,2 }}$ \\ ${ }^{1}$ Department of General Surgery, Capital Medical University Affiliated Beijing Friendship Hospital, Beijing, China \\ 2 National Clinical Research Center of Digestive Diseases, Beijing, China \\ 3 Department of Radiology, Capital Medical University Affiliated Beijing Friendship Hospital, Beijing, China \\ 4 Clinical Epidemiology and EBM Center, Capital Medical University Affiliated Beijing Friendship Hospital, Beijing, China \\ Corresponding Authors: Zhenghan Yang, Zhongtao Zhang \\ Email address: yangzhenghan@ccmu.edu.cn, zhangzhongtao@ccmu.edu.cn
}

Background: microRNAs (miRNAs) have been studied for their role in the early detection of several diseases. However, there is no current information on the systematic screening of serum-derived cisplatin resistance biomarkers in gastric cancer (GC).

Methods: Cisplatin-resistant GC cell lines were screened for dysregulated miRNAs using small RNA sequencing (sRNA-seq) and miRNAs were functionally annotated using bioinformatics analyses. Real-time quantitative polymerase chain reaction (RT-qPCR) was used to validate the miRNA-relative transcription levels in GC cells and in 74 GC patients. We analyzed the associations between the clinical characteristics of the patients and their miRNA expression. Receiver operating characteristic (ROC) analysis was used to evaluate the diagnostic value for serum-derived cisplatin resistance.

Results: 7 miRNAs were identified from 35 differentially expressed miRNAs between the MGC803/DDP and MGC803 cells in a public database. We found four miRNA candidates (miR-9-3p, miR-9-5p, miR-146a-5p, and miR-433-3p) that were significantly associated with chemotherapy responses in GC cells and patients. miR-9-5p (AUC $=0.856,95 \% \mathrm{Cl}: 0.773-0.939, p<0.0001$ ) and a combined group (miR-9-5p + miR-9-3p + miR-433-3p) (AUC = 0.915, 95\%Cl: 0.856-0.975, $P<0.0001)$ distinguished chemoresistant GC patients from chemosensitive GC patients.

Conclusions: Our study reveals the potential therapeutic use of 2 serum-based biomarkers, miR-9-5p and a combined group (miR-9-5p + miR-9-3p + miR-433-3p), as indicators for the successful use of cisplatin in GC patients. 
2 Serum microRNAs as potential new biomarkers for cisplatin resistance in gastric cancer 3 patients

Lei Jin ${ }^{1,4}$, Nan Zhang ${ }^{2,4}$, Qian Zhang ${ }^{3,4}$, Guoqian Ding ${ }^{1,4}$, Zhenghan Yang ${ }^{2,4}$ and Zhongtao Zhang ${ }^{1,4}$

1Department of General Surgery, Beijing Friendship Hospital, Capital Medical University, Beijing, China

2Department of Radiology, Beijing Friendship Hospital, Capital Medical University, Beijing, China

${ }^{3}$ Clinical Epidemiology and EBM Center, Beijing Friendship Hospital, Capital Medical University, Beijing, China

${ }^{4}$ National Clinical Research Center of Digestive Diseases, Beijing, China

Corresponding Author:

Zhongtao Zhang 1,4

95 Yongan Road, Xicheng District, Beijing, 100050, China

Email address: zhangzhongtao@ccmu.edu.cn

Zhenghan Yang 2,4

95 Yongan Road, Xicheng District, Beijing, 100050, China

Email address: yangzhenghan@ccmu.edu.cn

\section{Abstract}

Background: microRNAs (miRNAs) have been studied for their role in the early detection of several diseases. However, there is no current information on the systematic screening of serum-derived cisplatin resistance biomarkers in gastric cancer (GC).

Methods: Cisplatin-resistant GC cell lines were screened for dysregulated miRNAs using small RNA sequencing (sRNA-seq) and miRNAs were functionally annotated using bioinformatics analyses. Real-time quantitative polymerase chain reaction (RT-qPCR) was used to validate the miRNA-relative transcription levels in GC cells and in 74 GC patients. We analyzed the associations between the clinical characteristics of the patients and their miRNA expression. Receiver operating characteristic (ROC) analysis was used to evaluate the diagnostic value for serum-derived cisplatin resistance. 
35 Results: 7 miRNAs were identified from 35 differentially expressed miRNAs between the MGC803/DDP and MGC803 cells in a public database. We found four miRNA candidates (miR9-3p, miR-9-5p, miR-146a-5p, and miR-433-3p) that were significantly associated with chemotherapy responses in GC cells and patients. miR-9-5p (AUC = 0.856, 95\%Cl: 0.7730.939, $p<0.0001)$ and a combined group (miR-9-5p + miR-9-3p + miR-433-3p) (AUC = 0.915, 95\% Cl: 0.856-0.975, $P<0.0001)$ distinguished chemoresistant GC patients from chemosensitive GC patients.

Conclusions: Our study reveals the potential therapeutic use of 2 serum-based biomarkers, miR-9-5p and a combined group (miR-9-5p + miR-9-3p + miR-433-3p), as indicators for the successful use of cisplatin in GC patients.

\section{Introduction}

47 Gastric cancer is the fourth most commonly diagnosed malignant cancer and the second leading cause of tumor-related deaths worldwide (1). Surgery combined with chemotherapy is an emerging and treatment for GC.

Cisplatin (DDP) is recognized as a first-line chemotherapy drug for GC patients in a progressive stage of the disease. The current long-term survival rate of GC patients is poor due to the high prevalence of drug-resistance, metastasis, and recurrence (2). DDP resistance in most patients is inevitable and results in failed treatment, while the long-term use and repeated administration of DDP leads to severe side effects (3). The development of novel biomarkers for chemotherapy-resistant GC is critical for improving the prognostic efficacy in patients. miRNAs are a discovered class of small noncoding RNAs containing 19-25 nucleotides (4). miRNAs have been identified in many biofluids, including serum, plasma, and urine (5), suggesting that circulating miRNAs could be used as minimally invasive biomarkers for cancer and other diseases (5-7). The use of biomarkers is noninvasive, more comfortable than 
60 62 63 64 65 66

\section{Materials \& Methods}

75 Cell culture patients.

endoscopic examination, and may reflect the heterogeneity of the disease. Dysregulation of miRNAs may lead to DDP resistance in many tumors $(8,9)$, but the role of miRNAs in the chemo-response of GC is not fully understood.

miRNAs in human tumor serum specimens may act as markers to predict the treatment sensitivity and prognosis in many cancer types (10-12). Emerging evidence reveals that specific miRNAs in serum specimens may aid in the early diagnosis of malignancies $(13,14)$ and in the determination of the survival prognosis for cancer patients after surgery (15). However, it is not understood how specific miRNAs in serum might predict a chemotherapy response in GC

Small RNA-sequencing was used to analyze the microRNA profiles in GC cells to find the dysregulated miRNAs. RT-qPCR and correlation analyses were used to validate the results of sRNA-seq, which was used to select the miRNA candidates. We also attempted to develop specific biomarkers to determine the chemotherapy response of GC.

The MGC803 cell line was obtained from the Chinese Academy of Medical Sciences (Beijing,

77 China). DDP-resistant GC cells were established by culturing MGC803 cells in a continuous 78 stepwise fashion with gradually increasing concentrations of DDP from 0.05 to $5 \mu \mathrm{g} / \mathrm{ml}$ over a 79 period of 15 months. This resulted in a shift towards cell proliferation and an apoptosis 80 phenotype $(16,17)$. The cell line was continuously cultured with $2 \mu \mathrm{g} / \mathrm{ml}$ DDP to maintain its resistance. These two cell lines were cultured following standard culturing procedures and identified by short tandem repeat (STR) profile data compared with the ATCC, DSMZ, or JCRB databases (Cobioer Biosciences Co., Ltd, Los Altos, CA). 


\section{Patient enrollment and ethics statement}

86 Serum specimens were collected from 74 GC patients who met the following criteria: (1)

87 histologically confirmed gastric adenocarcinoma; (2) DDP-based chemotherapy used as first-

88 line treatment; (3) received at least 2 cycles of neoadjuvant chemotherapy or palliative

89 treatment; (4) chemotherapy efficacy could be evaluated by unenhanced and enhanced

90 computed tomography (CT) after 2 or 3 cycles; (5) serum samples were collected before the

91 first chemotherapy. All patients provided informed consent prior to the collection of a blood

92 sample. This study was approved by the Ethics Committee of Beijing Friendship Hospital,

93 Capital Medical University and met the ethical requirements of the Declaration of Helsinki.

94 Prechemotherapy serum samples were collected between January 2015 and January 2019

95 from patients who received neoadjuvant chemotherapy or palliative treatment. The serum

96 samples were collected and processed following the standard operating procedure of the Early

97 Detection Research Network. The chemotherapy principle was executed according to the 98 guidelines of the American Society of Clinical Oncology (ASCO). The chemotherapy response effect was evaluated according to $\mathrm{CHOl}$ 's principle (18). Treatment resistance was evaluated by the existing progressive disease $(P D)$ in $<3$ treatment cycles, and the treatment response was evaluated by the existing complete response (CR), stable disease (SD), or partial response (PR) lasting for 2 or 3 cycles. The chemotherapy response-sensitive group included CR and PR patients, whereas the chemotherapy response-resistant group included SD and PD patients.

\section{RNA extraction}

106 Total RNA was isolated from the GC cells using Trizol (Invitrogen, USA). The same amount of

107 Caenorhabditis elegans cel-39-3p miRNA was spiked into each serum sample as an external 108 calibration for RNA extraction, reverse transcription, and miRNA amplification. Total RNA was 109 extracted and purified from serum using the miRNeasy Serum/Plasma kit (Qiagen, cat. 217184). 
110 The quantity and integrity of the RNA yield was assessed using the Qubit2.0 fluorometer (Life

111 Technologies, USA) and Agilent 2200 TapeStation (Agilent Technologies, USA), separately.

112

113 sRNA-seq and data analysis

114 Total RNA (1 $\mu \mathrm{g})$ of GC cells were used to prepare small RNA libraries by NEBNext Multiplex

115 Small RNA Library Prep Set for Illumina (NEB, USA) according to the manufacturer's

116 instructions. The libraries were sequenced by HiSeq 2500 (Illumina, USA) with single-end 50 bp

117 reads at RiboBio Co. Ltd (RiboBio, China). Raw reads were processed by FastQC to get clean

118 reads by filtering out those containing adapter, poly ' $N$ ', were of low quality, or were smaller than

$11917 \mathrm{nt}$ reads. Mapping reads were obtained by mapping clean reads to the reference genome

120 from the BWA software. miRDeep2 was used to identify known mature miRNA based on

121 miRBase21 (www.miRBase.org) and to predict novel miRNA. The databases of Rfam12.1

122 (www.rfam.xfam.org) and piRNABank (www.pirnabank.ibab.ac.in) were used to identify

123 ribosomal RNA (rRNA), transfer RNA (tRNA), small nuclear RNA (snRNA), small nucleolar RNA

124 (snoRNA), and piwi-interacting RNA (piRNA) by BLAST. miRNA expressions were calculated in

125 RPM (reads per million) values (RPM = (number of reads mapping to miRNA/number of reads in

126 clean data $\left.) \times 10^{6}\right)$. The expression levels were normalized by RPM [(number of reads mapping

127 to miRNA/number of reads in clean data) $\left.\times 10^{6}\right]$.

128

129 Bioinformatics analysis

130 The differential expression between the two sample sets was calculated using the edgeR

131 algorithm according to the criteria of $\mid \log 2$ (Fold Change) $\mid \geqslant 1$ and $P$-value $<0.05$. TargetScan, 132 miRDB, miRTarBase, and miRWalk were used to predict the target genes of selected miRNA.

133 Patients with different expression levels of miRNAs were determined to have shorter or longer 134 overall survival (OS) times for 10-year OS using the Kaplan-Meier survival curve data according 
135 to the Kaplan-Meier Plotter database (http://kmplot.com) $(19,20)$, the OncoLnc database

136 (http://www.oncolnc.org/) (21), and the OncomiR database (http://www.oncomir.org/) (22).

137

138 RT-qPCR

139 The primers for miRNA detection were purchased from FulenGene Company (Guangzhou,

140 China) and their sequence is listed in Table 1. Total RNA was reverse transcribed using the All-

141 in-One $^{T M}$ miRNA RT-qPCR Kit (GeneCopoeia, USA) for miRNA analysis. The All-in-One ${ }^{\mathrm{TM}}$

142 miRNA RT-qPCR Detection Kit (GeneCopoeia, USA) was used to measure miRNAs

143 quantitatively. All samples were normalized by the initial biofluid input volume used for RNA

144 extraction and were calibrated by the spike-in cel-39-3p to eliminate the minute bias caused by

145 different RNA isolation efficiencies and PCR efficiencies among samples. U6 or let-7g-5p was

146 used as an endogenous control to normalize the relative number of miRNAs. ABI 7500 real-time

147 fast PCR system (Applied Biosystems, USA) was used to achieve the relative quantitation of

148 miRNA expression and the data was analyzed using the affiliated software. The Ct attenuation

149 value of each type of miRNA in each of the serum samples was corrected by the internal

150 reference cel-39-3p and the two housekeeping genes, U6 and let-7g-5p. The $2^{-\Delta \Delta c t}$ method was

151 used to calculate the relative expression of each miRNA. Each sample per reaction was

152 performed in triplicate.

153

154 Statistical analysis

155 miRNA expression levels in the serum samples were divided into high and low groups taking the

156 median as the cutoff value by RT-qPCR. Statistical differences for in vitro experiments were

157 analyzed using student's unpaired $t$ tests. Associations between the clinical parameters of the

158 patients and their miRNA expression were analyzed using the Mann-Whitney test. The AUC

159 was used to assess the diagnostic accuracy of the predictors. Logistic regression was used to

160 develop a panel of combined biomarkers to predict the probability of GC chemotherapy

Peer] reviewing PDF | (2019:10:41928:1:2:NEW 18 Feb 2020) 
161 response. All data were expressed as mean \pm SD. Each experiment was repeated

162 independently at least three times. Quantitative data were analyzed and graphed using SPSS

163 23.0, MedCalc, and GraphPad Prism 7. Differences were considered to be significant at ${ }^{\star * * *} P<$

$1640.0001,{ }^{* \star *} P<0.001,{ }^{* *} P<0.01$, and ${ }^{*} P<0.05$.

165

166 Results

167 Analysis of sRNA-seq data

168 The results of sRNA-seq in MGC803/DDP cells (Figure 1A) and MGC803 cells (Figure 1B)

169 showed that the sRNA sequence included miRNA, tRNA, rRNA, snRNA, snoRNA, piRNA, and

170 other RNA. Thirty-five miRNAs were selected and intersected, of which 11 miRNAs were up-

171 regulated, and 24 down-regulated at higher levels in MGC803/DDP cells than the MGC803 cells

172 (Figure 1C). These 35 differentially expressed miRNAs in sRNA-seq data are shown in Table 2.

173

174 Bioinformatics analysis of selected miRNA

175 A large cohort analysis was conducted using Kaplan-Meier survival data according to the

176 Kaplan-Meier Plotter database in order to determine the role of the 35 differentially expressed

177 miRNAs as a potential prognostic factor. We observed that a total of seven differentially

178 expressed miRNAs showed statistical differences $(p<0.05)$ for 10 -year overall survival (OS)

179 (Table 2), which indicated that these miRNAs could be used as valid biomarkers for

180 chemotherapy response and overall survival. The differentially expressed miRNAs observed

181 were miR-9 (including miR-9-3p and miR-9-5, Figure 2A), miR-146a (Figure 2B), miR-370

182 (Figure 2C), miR-433 (Figure 2D), miR-519a (Figure 2E), and miR-522 (Figure 2F). TargetScan,

$183 \mathrm{miRDB}$, miRTarBase, and miRWalk were used to predict the target genes of the seven selected

184 miRNAs, including miR-9-3p (Figure 3A), miR-9-5p (Figure 3B), miR-146a-5p (Figure 3C), miR-

185 370-3p (Figure 3D), miR-433-3p (Figure 3E), miR-519a-5p (Figure 3F), and miR-522-5p (Figure

$1863 G$ ). Supplementary material from four different databases (Supplemental table 1) was added to

Peer] reviewing PDF | (2019:10:41928:1:2:NEW 18 Feb 2020) 
187 the common target genes of the 7 miRNAs (Figure 3). These 7 miRNAs were selected as 188 miRNA candidates.

190 Validation of sRNA-seq data by RT-qPCR analysis

191 GC cell results showed that the levels of miR-146a-5p, miR-519a-5p, and miR-522-5p were 192 significantly downregulated in MGC803/DDP cells. The levels of miR-9-3p, miR-9-5p, miR-370193 3p, and miR-433-3p were significantly upregulated in MGC803/DDP cells (Table 3, Figure 4A). 194 miR-519a-5p and miR-522-5p changed consistently with sRNA-seq, but the opposite results 195 were seen from the other five miRNAs. miR-519a-5p and miR-522-5p had the same primer and 196 exhibited the same result.

197

\section{Expression levels and functions of miRNAs in human GC clinical specimens}

RT-qPCR tests were run on the serum samples from 74 GC patients to identify 2 potential miRNAs, 2 housekeeping genes (U6 and let-7g-5p), and a spike-in cel-39-3p of the internal references.

The clinical parameter findings of 74 patients with neoadjuvant chemotherapy or palliative treatment are shown in Table 4. Using the median ratio as the cutoff for the relative miRNA expression (7 miRNA candidates; Fold Change $=0$ ) in serum, patients were classified into two groups: low miRNA (7 miRNA candidates) and high miRNA (7 miRNA candidates). Clinical data was collected and efficacy was evaluated by CT in 2-3 cycles following chemotherapy. Independent measurements were made by 2 radiologists according to $\mathrm{CHOl}$ 's principle (18). Unenhanced-combined-enhanced CT was applied to accurately evaluate the tumor response using tumor density measurement. RT-qPCR analysis was used to detect miRNA (7 miRNA candidates) levels in 34 chemotherapy response-sensitive (CR and PR) and 40 chemotherapy

211 response-resistant (SD and PD) patients to validate miRNA (7 miRNA candidates) expression 212 levels. 
213 Our results proved that miR-9-3p expression obviously increased in the serum samples of 214 chemotherapy response-resistant GC patients; $67.5 \%$ (27 of 40 ) of the high miR-9-3p samples 215 showed DDP resistance ( $p<0.0001$, Figure 4B). miR-9-5p expression increased in the serum 216 samples of chemotherapy response-resistant GC patients; $72.5 \%$ (29 of 40 ) of the high miR-9$2175 p$ samples showed DDP resistance $(p<0.0001$, Figure $4 C)$. miR-146a-5p expression was 218 obviously induced in the serum samples of chemotherapy response-resistant GC patients; $21962.5 \%$ (25 of 40$)$ of the low miR-146a-5p samples showed DDP resistance $(p<0.0001$, Figure 220 4D). miR-433-3p expression increased in the serum samples of chemotherapy response221 resistant GC patients; $67.5 \%$ (27 of 40 ) of the high miR-433-3p samples showed DDP 222 resistance $(p<0.0001$, Figure 4E). However, there was no significant difference between the 223 relative levels of miR-370-3p ( $p=0.0611$, Figure $4 F)$, miR-519a-5p $(p=0.4028$, Figure $4 \mathrm{G})$, and $224 \operatorname{miR}-522-5 p(p=0.4028$, Figure $4 \mathrm{H})$ in the serum of 34 chemotherapy response-sensitive gastric 225 cancer samples and 40 chemotherapy response-resistant gastric cancer samples.

226 Our results indicate that miR-9-3p, miR-9-5p, miR-146a-5p, and miR-433-3p may act as 227 potential new biomarkers for the chemotherapy response of DDP treatment. The levels of miR228 9-3p, miR-9-5p, miR-146a-5p, and miR-433-3p were not related to gender and age (Table 4).

229

230

Receiver operating characteristic (ROC) analysis of miRNAs

231 Four miRNAs distinguished the GC chemotherapy response-resistant (SD+PD) group from the 232 GC chemotherapy response-sensitive group (CR+PR). The results for the area under the 233 curves (AUC), standard deviation (SD), 95\% confidence intervals (CI), $P$-values, sensitivities 234 (SE), and specificities (SP) of these miRNAs were as follows (Table 5): miR-9-3p (AUC = 0.824, 235 Figure 5A), miR-9-5p (AUC = 0.856, Figure 5B), miR-146a-5p $(A \cup C=0.799$, Figure 5C $)$ and $236 \mathrm{miR}-433-3 p(A \cup C=0.838$, Figure 5D), respectively. These four miRNAs provided promising 237 AUC values for differentiating the GC chemotherapy response-resistant groups from the GC 238 chemotherapy response-sensitive groups. 
239 Two of the four candidate miRNAs were combined in a logistic model, with a significantly

240 improved performance compared with the individual miRNA, determined by (Table 5): miR-9-3p

$241+$ miR-9-5p $(A U C=0.889$, Figure $6 A)$. The risk score factors $(R S F)$ were calculated as $0.731 \times$

$242 \mathrm{miR}-9-3 p+0.586 \times \operatorname{miR}-9-5 p+0.192 ; \mathrm{miR}-9-3 p+\operatorname{miR}-146 a-5 p(A \cup C=0.903$, Figure 6B), and

$243 \mathrm{RSF}=0.800 \times \mathrm{miR}-9-3 p-0.772 \times \mathrm{miR}-146 a-5 p+0.392 ; \mathrm{miR}-9-3 p+\operatorname{miR}-433-3 p(A U C=0.865$,

244 Figure 6C), and RSF $=0.574 \times \operatorname{miR}-9-3 p+0.684 \times \operatorname{miR}-433-3 p+0.173 ; \mathrm{miR}-9-5 p+\mathrm{miR}-146 a-$

$2455 p(A U C=0.901$, Figure 6D), and RSF $=0.575 \times \operatorname{miR}-9-5 p-0.746 \times \operatorname{miR}-146 a-5 p+0.345$

$246 \mathrm{miR}-9-5 p+\operatorname{miR}-433-3 p(A \cup C=0.898$, Figure 6E), and RSF $=0.551 \times \operatorname{miR}-9-5 p+0.847 \times \mathrm{miR}-$

$247433-3 p+0.264 ;$ miR-146a-5p + miR-433-3p (AUC = 0.885, Figure 6F $)$, and RSF $=-0.637 \times$

248 miR-146a-5p + $0.802 \times \operatorname{miR}-433-3 p+0.327$

249 Three or more of the four candidate miRNAs were combined resulting in a significantly improved 250 performance compared with the individual miRNA. (Table 5 miR-9-3p + miR-9-5p + miR-146a$2515 p)(A \cup C=0.930$, Figure 7A). The RSF was calculated as $0.690 \times$ miR-9-3p $+0.515 \times$ miR-9$2525 p-0.738 \times \operatorname{miR}-146 a-5 p+0.386 ;$ miR-9-3p + miR-9-5p + miR-433-3p (AUC = 0.915, Figure 253 7B), $R S F=0.514 \times$ miR-9-3p + $0.526 \times \operatorname{miR}-9-5 p+0.614 \times \operatorname{miR}-433-3 p+0.240 ; \mathrm{miR}-9-3 p+$ 254 miR-146a-5p + miR-433-3p (AUC = 0.918, Figure 7C), RSF $=0.646 \times$ miR-9-3p $-0.700 \times \mathrm{miR}-$ 255 146a-5p + $0.504 \times$ miR-433-3p + 0.388; miR-9-5p + miR-146a-5p + miR-433-5p (AUC = 0.926, 256 Figure 7D), RSF = $0.521 \times \operatorname{miR}-9-5 p-0.650 \times \operatorname{miR}-146 a-5 p+0.655 \times \operatorname{miR}-433-3 p+0.376$; 257 miR-9-3p + miR-9-5p + miR-146a-5p + miR-433-3p (AUC = 0.937, Figure 7E), RSF $=0.540 \times$ $258 \mathrm{miR}-9-3 p+0.480 \times \mathrm{miR}-9-5 p-0.681 \times \mathrm{miR}-146 a-5 p+0.393 \times \mathrm{miR}-433-3 p+0.398$

260 Pairwise comparison of ROC curves

261 MedCalc software was used to display the data for the different ROC curves and the results of 262 pairwise comparison of all ROC curves (difference between the areas (DBA), standard error 263 (SE), 95\% confidence interval for the difference (CI), and P-value) (Table 6). 
264 The ROC curve of miR-9-5p had the best AUC value of the four miRNA candidates. A

265 comparison between the combined group (any different miRNA combination, which contained

266 miR-9-5p), and the miR-9-5p group showed that the two compared areas were significantly

267 different $(p=0.026$, Figure $8 A)$. The compared areas of the combined group (miR-9-5p + miR-9-

$2683 p+\operatorname{miR}-433-3 p)$ and the miR-9-5p group were significantly different $(p=0.045$, Figure $8 \mathrm{~B})$. The

269 compared areas of the combined group (miR-9-5p + miR-146a-5p + miR-433-3p) and the miR-

$2709-5 p$ group were significantly different $(p=0.034$, Figure $8 C)$ and the compared areas of the

271 combined group (miR-9-5p + miR-9-3p + miR-146a-5p + miR-433-3p) and the miR-9-5p group

272 were also significantly different ( $p=0.016$, Figure 8D). However, the compared areas of the other

273 combined group and the miR-9-5p group were not significantly different $(P>0.05)$.

274

275 Validate selected miRNAs in public database

276 To determine the role of miR-9-3p, miR-9-5p, miR-146a-5p, miR-433-3p, or their combination as

277 a potential prognostic factor, a large cohort analysis was conducted using Kaplan-Meier

278 survival data according to the Kaplan-Meier Plotter database, the OncoLnc database, and the

279 OncomiR database (Table 7).

280 We observed that patients with higher miR-9-3p levels had shorter OS times than those with

281 lower miR-9-3p levels in the Oncolnc database $(p=0.00836$, Figure $9 A)$ and the OncomiR

282 database $(p=0.06643$, Figure 9B). Patients with higher miR-9-5p levels had shorter OS times

283 than those with lower miR-9-5p levels in the OncoLnc database $(p=0.00181$, Figure $9 \mathrm{C})$ and the

284 OncomiR database ( $p=0.0044$, Figure 9D). Patients with higher miR-433-3p levels had shorter

285 OS times than those with lower miR-433-3p levels in the OncoLnc database $(p=0.0311$, Figure

$2869 \mathrm{E})$ and the OncomiR database $(p=0.05651$, Figure 9F). These observations indicate that the

287 high expression of miR-9-3p, miR-9-5p, or miR-433-3p are a valid biomarker for

288 chemoresistance and poor survival, especially miR-9-5p. 
289 Patients with higher levels of the combined group (miR-9-5p + miR-9-3p) had shorter OS times 290 than those with lower combined levels in the OncomiR database $(p=0.003421$, Figure 10A).

291 Patients with higher levels of the combined group (miR-9-5p + miR-433-3p) had shorter OS

292 times than those with lower combined levels in the OncomiR database $(p=0.01428$, Figure $10 \mathrm{~B})$.

293 Patients with higher levels of the combined group (miR-9-5p + miR-9-3p + miR-433-3p) had

294 shorter OS times than those with lower combined levels in the OncomiR database $(p=0.008066$,

295 Figure 10C). Patients with higher levels of the combined group (miR-9 + miR-433) had shorter

296 OS times than those with lower combined levels in the Kaplan-Meier Plotter database

$297(p=0.0047$, Figure 10D). These observations indicate that higher levels of the combined groups, 298 especially the combined group (miR-9-5p + miR-9-3p + miR-433-3p) are a valid biomarker for 299 chemoresistance and poor survival.

300

301

\section{Discussion}

302 DDP resistance is a barrier to the effective treatment of GC. The identification of unique

303 biomarkers for drug resistance can help to accurately diagnose and treat GC.

304 The involvement of non-coding nucleic acids, such as miRNAs in response to DDP treatment, is 305 not well understood. miRNAs play vital roles in the progression and development of tumors, and 306 may be correlated with resistance to chemotherapeutics in tumor cells (23-25). The downregulation of miR-21 has been shown to alter survival rates by increasing DDP sensitivity in GC cells (26).

Liquid biopsies can identify markers from blood and various body fluids and are minimally invasive, safe, economical, and convenient versus the use of more invasive tests or biopsies for

311 the diagnosis of GC (27). Liquid biopsies are suitable for screening a wide range of people,

312 which can improve the diagnosis and treatment of GC. Several studies have been conducted on

313 the use of miRNAs in GC patients related to the occurrence, development, diagnosis, treatment, 314 and prognosis of the disease. Fang et al. (28) reported that certain carcinogenesis-related 
315 miRNAs (miR-10b, miR-21, miR-223, and miR-338) and tumor suppressor miRNAs (miR-30a$3165 p$, miR-126, and let-7a) can be used as prognosis markers in GC patients. Numerous studies

317 have reported on the use of combinations of circulating miRNAs for greater diagnostic accuracy, 318 which is indicated by an area under the ROC curve larger than $0.8(28,29)$.

319 Relatively few studies have reported the application of miRNAs in the diagnosis and screening 320 of the chemotherapy response of GC. In this study, the differences in the expression profiles of 321 miRNAs in chemotherapy response-sensitive and chemotherapy response-resistant GC cells 322 were established using sRNA-seq and the potential miRNAs were screened using 323 bioinformatics analyses.

324 Our results showed that miR-9-3p, miR-9-5p, and miR-433-3p were significantly up-regulated 325 and miR-146a-5p was significantly down-regulated in the MGC803/DDP cells and in 326 chemotherapy response-resistant GC patients. While some of these miRNAs are considered to be diagnostic or prognostic biomarkers in GC, ours is the first study to explore their potential use in determining the chemotherapy response in GC patients.

Numerous studies have shown that a combination of multiple miRNAs could more easily identify early-stage CC patients than a single miRNA biomarker $(6,29)$. We combined the three miRNAs in our study (miR-9-3p, miR-9-5p and miR-433-3p) to achieve an AUC of 0.915. The equation used in this determination was risk score factor $(R S F)=0.514 \times$ miR-9-3p $+0.526 \times$ miR-9-5p $+0.614 \times$ miR-433-3p +0.240 . There was a significant difference between the two compared areas of the combined group (miR-9-3p + miR-9-5p + miR-433-3p) and the miR-9-5p group ( $p=0.045)$, whereas the two compared areas of the other combined group and the miR-9-

$3365 p$ group was not significantly different $(p>0.05)$. A large cohort analysis was conducted using

337 Kaplan-Meier survival data according to the Kaplan-Meier Plotter database, the OncoLnc database, and the OncomiR database to determine the role of miR-9-3p, miR-9-5p, miR-433-3p or their combination as a potential prognostic factor. The results indicate that the high 
340

341

342

343

344

345

346

347

348

349

350

351

352

353

354

355

356

357

358

359

360

361

362

363

364

365

366

367

368

369

370

371

372

373

374

expression of miR-9-5p and the combined group (miR-9-5p + miR-9-3p + miR-433-3p) can be

used as a valid biomarker for chemoresistance and poor survival. Serum-derived miRNAs have

the potential to be used as novel noninvasive tumor markers for the chemotherapy response in

GC patients.

Previous studies have shown that miR-9-5p expression may act as a potential tumor suppressor gene and is closely related to the malignant progression of GC (30). miR-9-3p may play an important role in tumor invasion and have potential effects on the prognosis of gastric cancer (31). miR-433-3p may function as a potential diagnostic marker and therapeutic target for glioma (32). These miRNAs serve as biomarkers for tumors and tumor progression.

\section{Conclusions}

In conclusion, we reported on the potential for 2 serum-based biomarkers, miR-9-5p and a combined group (miR-9-5p + miR-9-3p + miR-433-3p), to predict the therapeutic benefit of DDP for GC patients. Additional clinical samples should be collected to validate these serum biomarkers.

\section{Acknowledgements}

We thank Biobank Resource and Clinical Data of Beijing Friendship Hospital, Capital Medical University for their collection of serum samples and their data processing services.

\section{References}

1 Siegel RL, Miller KD and Jemal A: Cancer statistics, 2019. CA Cancer J Clin 69(1): 7-34, 2019. PMID, DOI: 10.3322/caac. 21551

2 Zong L, Abe M, Seto Y and Ji J: The challenge of screening for early gastric cancer in china. The Lancet 388(10060): 2606, 2016. PMID, DOI: 10.1016/s0140-6736(16)32226-7

3 Kovalchuk O, Filkowski J, Meservy J, Inytskyy Y, Tryndyak VP, Chekhun VF and Pogribny IP: Involvement of microrna-451 in resistance of the mcf-7 breast cancer cells to chemotherapeutic drug doxorubicin. Mol Cancer Ther 7(7): 2152-2159, 2008. PMID, DOI: 10.1158/1535-7163.MCT-08-0021

4 Bartel, DP: MicroRNAs: genomics, biogenesis, mechanism, and function. Cell 116(2): 281-97, 2004. PMID, DOI: 10.1016/S0092-8674(04)00045-5

5 Etheridge A, Lee I, Hood L, Galas D and Wang K: Extracellular microrna: A new source of biomarkers. Mutation Research/Fundamental and Molecular Mechanisms of Mutagenesis 717(1-2): 85-90, 2011. PMID, DOI: 10.1016/j.mrfmmm.2011.03.004

6 Vychytilova-Faltejskova P, Radova L, Sachlova M, Kosarova Z, Slaba K, Fabian P, Grolich T, Prochazka V, Kala Z, Svoboda M, Kiss I, Vyzula R and Slaby O: Serum-based microrna signatures in early diagnosis and prognosis 
375

376

377

378

379

380

381

382

383

384

385

386

387

388

389

390

391

392

393

394

395

396

397

398

399

400

401

402

403

404

405

406

407

408

409

410

411

412

413

414

415

416

417

418

419

420

421

422

423

424

425

426

427

prediction of colon cancer. Carcinogenesis 37(10): 941-950, 2016. PMID, DOI: 10.1093/carcin/bgw078

7 Schultz NA, Dehlendorff C, Jensen BV, Bjerregaard JK, Nielsen KR, Bojesen SE, Calatayud D, Nielsen SE, Yilmaz M, Hollander NH, Andersen KK and Johansen JS: Microrna biomarkers in whole blood for detection of pancreatic cancer. JAMA 311(4): 392-404, 2014. PMID, DOI: 10.1001/jama.2013.284664

8 Sorrentino A, Liu CG, Addario A, Peschle C, Scambia G and Ferlini C: Role of micrornas in drug-resistant ovarian cancer cells. Gynecol Oncol 111(3): 478-486, 2008. PMID, DOI: 10.1016/j.ygyno.2008.08.017

9 Yu ZW, Zhong LP, Ji T, Zhang P, Chen WT and Zhang CP: Micrornas contribute to the chemoresistance of cisplatin in tongue squamous cell carcinoma lines. Oral Oncol 46(4): 317-322, 2010. PMID, DOI: 10.1016/j.oraloncology.2010.02.002

10 Ueda T, Volinia S, Okumura H, Shimizu M, Taccioli C, Rossi S, Alder H, Liu C-g, Oue N, Yasui W, Yoshida K, Sasaki $\mathrm{H}$, Nomura S, Seto $\mathrm{Y}$, Kaminishi M, Calin GA and Croce CM: Relation between microrna expression and progression and prognosis of gastric cancer: A microrna expression analysis. The Lancet Oncology 11(2): 136-146, 2010. PMID, DOI: 10.1016/S1470-2045(09)70343-2

11 Goswami RS, Atenafu EG, Xuan Y, Waldron L, Reis PP, Sun T, Datti A, Xu W, Kuruvilla J, Good DJ, Lai R, Church AJ, Lam WS, Baetz T, Lebrun DP, Sehn LH, Farinha P, Jurisica I, Bailey DJ, Gascoyne RD, Crump M and KamelReid S: Microrna signature obtained from the comparison of aggressive with indolent non-hodgkin lymphomas: Potential prognostic value in mantle-cell lymphoma. J Clin Oncol 31(23): 2903-2911, 2013. PMID, DOI: 10.1200/JCO.2012.45.3050

12 Hur K, Toiyama Y, Schetter AJ, Okugawa Y, Harris CC, Boland CR and Goel A: Identification of a metastasis-specific microrna signature in human colorectal cancer. J Natl Cancer Inst 107(3), 2015. PMID: 4334826, DOI: $10.1093 /$ jnci/dju492

13 Masuda S and Izpisua Belmonte JC: Re: Serum mir-21 as a diagnostic and prognostic biomarker in colorectal cancer. J Natl Cancer Inst 106(3): djt457, 2014. PMID, DOI: 10.1093/jnci/djt457

14 Lin X-J, Chong Y, Guo Z-W, Xie C, Yang X-J, Zhang Q, Li S-P, Xiong Y, Yuan Y, Min J, Jia W-H, Jie Y, Chen M-S, Chen $M-X$, Fang J-H, Zeng C, Zhang $Y$, Guo R-P, Wu Y, Lin G, Zheng L and Zhuang S-M: A serum microrna classifier for early detection of hepatocellular carcinoma: A multicentre, retrospective, longitudinal biomarker identification study with a nested case-control study. The Lancet Oncology 16(7): 804-815, 2015. PMID, DOI: 10.1016/s1470-2045(15)00048-0

15 Hu Z, Chen X, Zhao Y, Tian T, Jin G, Shu Y, Chen Y, Xu L, Zen K, Zhang C and Shen H: Serum microrna signatures identified in a genome-wide serum microrna expression profiling predict survival of non-small-cell lung cancer. J Clin Oncol 28(10): 1721-1726, 2010. PMID, DOI: 10.1200/JCO.2009.24.9342

16 Hong W. S., Saijo N., Sasaki Y., Minato K., Nakano H., Nakagawa K., Fujiwara Y., Nomura K., and Twentyman P. R. 1988. Establishment and characterization of cisplatin-resistant sublines of human lung cancer cell lines. Int J Cancer 41:462-467. 10.1002/ijc.2910410325

17 Yu D. S., Ma C. P., and Chang S. Y. 2000. Establishment and characterization of renal cell carcinoma cell lines with multidrug resistance. Urological research 28:86-92. 10.1007/s002400050143

18 Choi H, Charnsangavej C, Faria SdC, Tamm EP, Benjamin RS, Johnson MM, Macapinlac HA and Podoloff DA: Ct evaluation of the response of gastrointestinal stromal tumors after imatinib mesylate treatment: $A$ quantitative analysis correlated with fdg pet findings. American Journal of Roentgenology 183(6): 16191628, 2004. PMID, DOI: 10.2214/ajr.183.6.01831619

19 Szász AM, Lánczky A, Nagy Á, Förster S, Hark K, Green JE, Boussioutas A, Busuttil R, Szabó A and Győrffy B: Crossvalidation of survival associated biomarkers in gastric cancer using transcriptomic data of 1,065 patients. Oncotarget 7(31): 49322-49333, 2016. PMID, DOI: 10.18632/oncotarget.10337

20 Nagy A, Lanczky A, Menyhart $O$ and Gyorffy B: Validation of mirna prognostic power in hepatocellular carcinoma using expression data of independent datasets. Sci Rep 8(1): 9227, 2018. PMID: 6003936, DOI: 10.1038/s41598-018-27521-y

21 Anaya J: OncoLnc: linking TCGA survival data to mRNAs, miRNAs, and IncRNAs. PeerJ Computer Science 2:e67, 2016. PMID, DOI: $10.7717 /$ peerj-cs.6710.7287/peerj.preprints.1780v1

22 Wong NW, Chen Y, Chen S and Wang X: Oncomir: An online resource for exploring pan-cancer microrna dysregulation. Bioinformatics 34(4): 713-715, 2018. PMID: 5860608, DOI: 10.1093/bioinformatics/btx627

23 Xia L, Zhang D, Du R, Pan Y, Zhao L, Sun S, Hong L, Liu J and Fan D: Mir-15b and mir-16 modulate multidrug resistance by targeting bcl2 in human gastric cancer cells. Int J Cancer 123(2): 372-379, 2008. PMID, DOI: $10.1002 / \mathrm{ijc} .23501$

Peer) reviewing PDF | (2019:10:41928:1:2:NEW 18 Feb 2020) 
428

429

430

431

432

433

434

435

436

437

438

439

440

441

442

443

444

445

446

447

448

449

450

451

452

24 Yang H, Kong W, He L, Zhao JJ, O'Donnell JD, Wang J, Wenham RM, Coppola D, Kruk PA, Nicosia SV and Cheng JQ: Microrna expression profiling in human ovarian cancer: Mir-214 induces cell survival and cisplatin resistance by targeting pten. Cancer Res 68(2): 425-433, 2008. PMID, DOI: 10.1158/0008-5472.CAN-07-2488

25 Li T, Li D, Sha J, Sun P and Huang Y: Microrna-21 directly targets marcks and promotes apoptosis resistance and invasion in prostate cancer cells. Biochem Biophys Res Commun 383(3): 280-285, 2009. PMID, DOI: 10.1016/j.bbrc.2009.03.077

26 Yang SM, Huang C, Li XF, Yu MZ, He Y and Li J: Mir-21 confers cisplatin resistance in gastric cancer cells by regulating pten. Toxicology 306(162-168, 2013. PMID, DOI: 10.1016/j.tox.2013.02.014

27 Tsujiura M, Ichikawa D, Konishi H, Komatsu S, Shiozaki A and Otsuji E: Liquid biopsy of gastric cancer patients: Circulating tumor cells and cell-free nucleic acids. World journal of gastroenterology 20(12): 3265-3286, 2014. PMID, DOI: 10.3748/wjg.v20.i12.3265

28 Fang Y, Shen H, Li H, Cao Y, Qin R, Long L, Zhu X, Xie C and Xu W: Mir-106a confers cisplatin resistance by regulating pten/akt pathway in gastric cancer cells. Acta Biochim Biophys Sin (Shanghai) 45(11): 963-972, 2013. PMID, DOI: 10.1093/abbs/gmt106

29 Ng EK, Chong WW, Jin H, Lam EK, Shin VY, Yu J, Poon TC, Ng SS and Sung JJ: Differential expression of micrornas in plasma of patients with colorectal cancer: A potential marker for colorectal cancer screening. Gut 58(10): 1375-1381, 2009. PMID, DOI: 10.1136/gut.2008.167817

30 Fan Y, Shi Y, Lin Z, Huang X, Li J, Huang W, Shen D, Zhuang G and Liu W: Mir-9-5p suppresses malignant biological behaviors of human gastric cancer cells by negative regulation of tnfaip8l3. Dig Dis Sci 64(10): 2823-2829, 2019. PMID, DOI: 10.1007/s10620-019-05626-2

31 Meng Q, Xiang L, Fu J, Chu X, Wang C and Yan B: Transcriptome profiling reveals mir-9-3p as a novel tumor suppressor in gastric cancer. Oncotarget 8(23): 37321-37331, 2017. PMID, DOI: 10.18632/oncotarget.16310

32 Sun S, Wang X, Xu X, Di H, Du J, Xu B, Wang Q and Wang J: Mir-433-3p suppresses cell growth and enhances chemosensitivity by targeting creb in human glioma. Oncotarget 8(3): 5057-5068, 2017. PMID, DOI: 10.18632/oncotarget.13789

Peer] reviewing PDF | (2019:10:41928:1:2:NEW 18 Feb 2020) 
Figure 1

Analysis for sRNA-seq data of MGC803/DDP and MGC803 groups.

(A, B)Pie charts showing the percentage of different kinds of sRNA to the mapped reads for MGC803 group (A) and MGC803/DDP group (B). (C)A heat map of the 35 diferentially expressed miRNAs in MGC803/DDP and MGC803 groups was showed. 

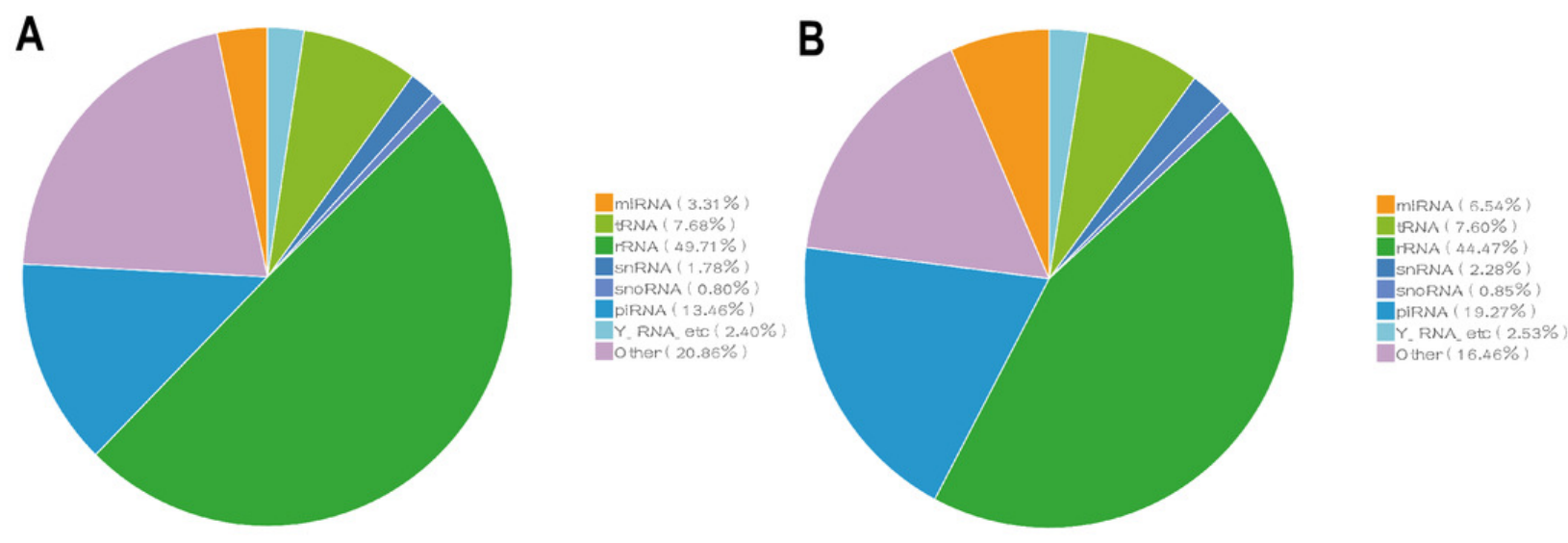

C
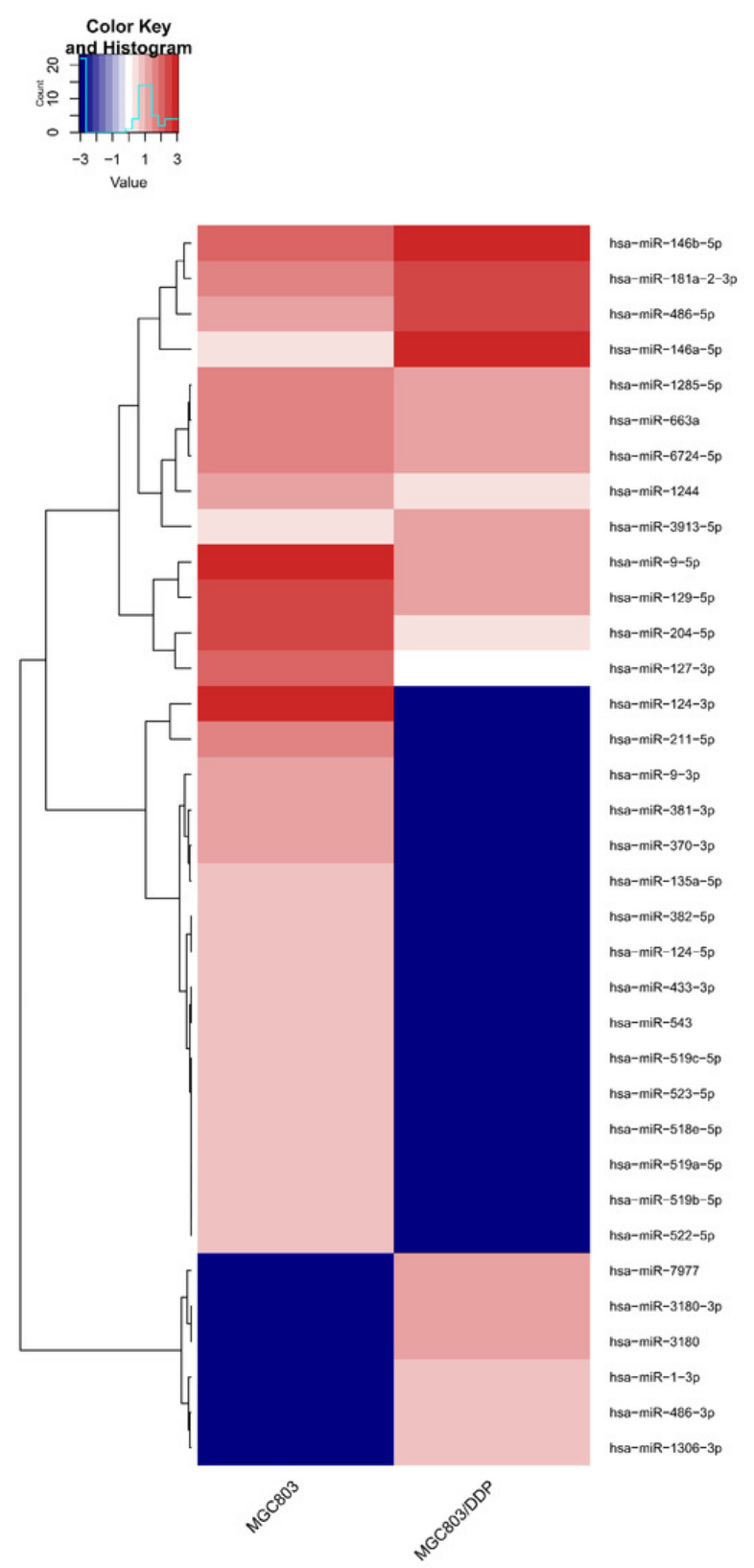
Figure 2

The role of 7 selected miRNAs was ascertained using Kaplan-Meier survival data according to Kaplan-Meier Plotter.

(A-F) Kaplan-Meier survival curves suggested that patients with high miR-9 (A), miR-370 (C), miR-433 (D), miR-519a (E) and miR-522 (F) levels had lower OS times for 10-year OS than those with low levels, and patients with high miR-146a (B) levels had higher OS times for 10year OS than those with those low levels. 
A
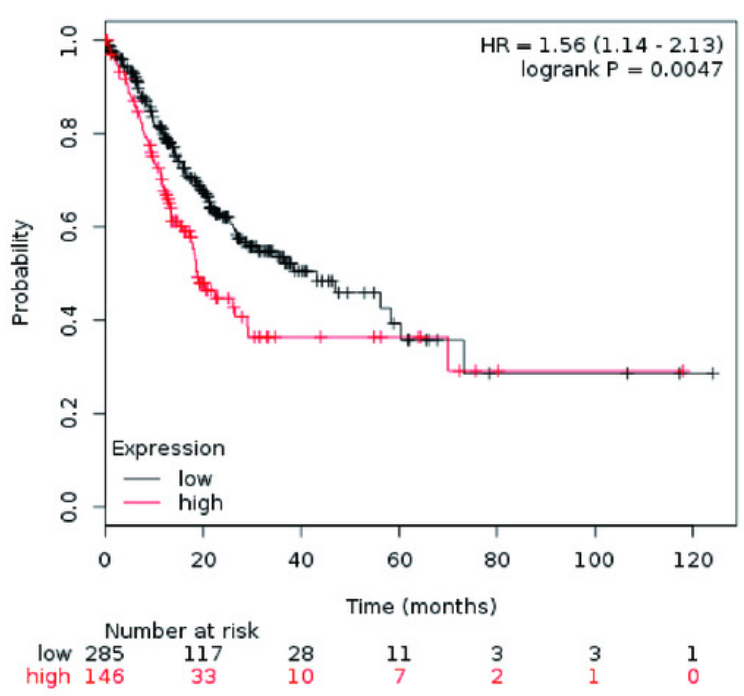

C
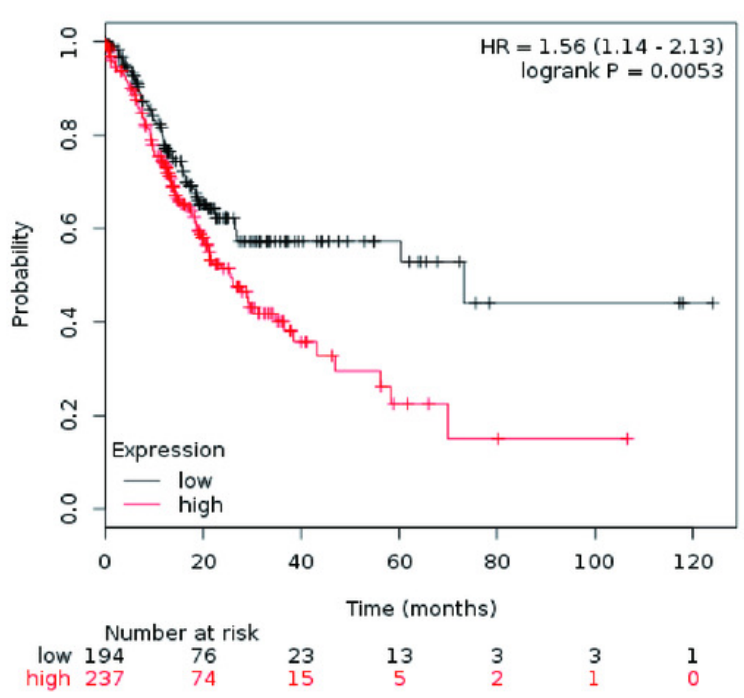

E

hsa-mir-519a

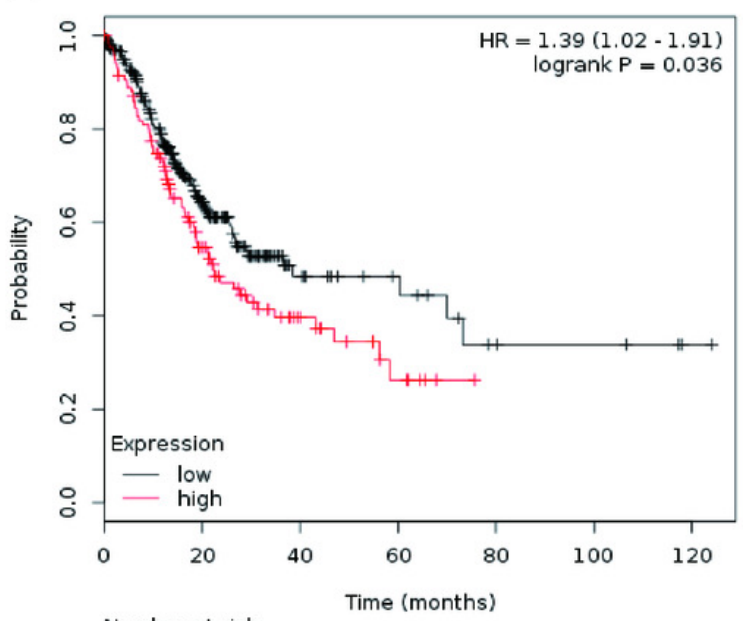

\begin{tabular}{ccccccc}
\multicolumn{7}{c}{ Number at risk } \\
low 312 & 102 & 20 & 12 & 5 & 4 & 1 \\
high 119 & 48 & 18 & 6 & 0 & 0 & 0
\end{tabular}
B

hsa-mir-146a

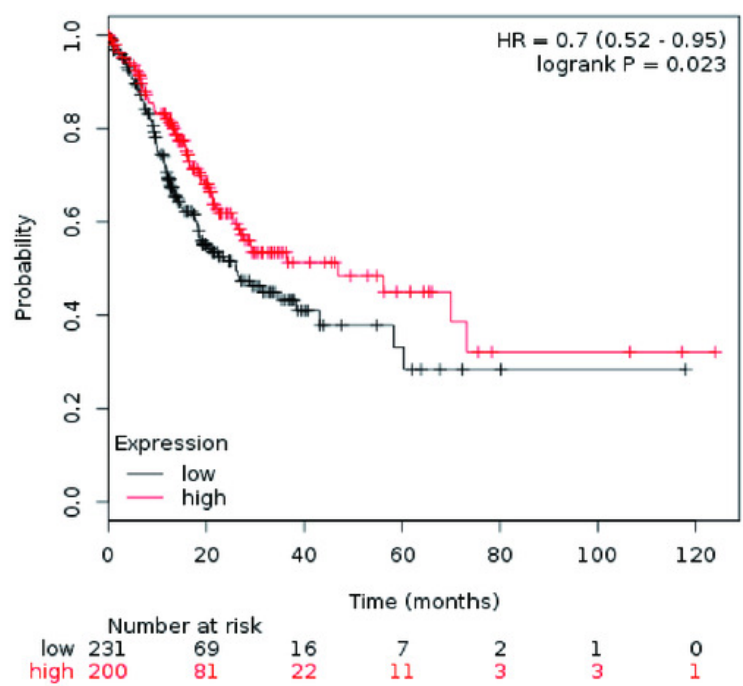

D

hsa-mir-522

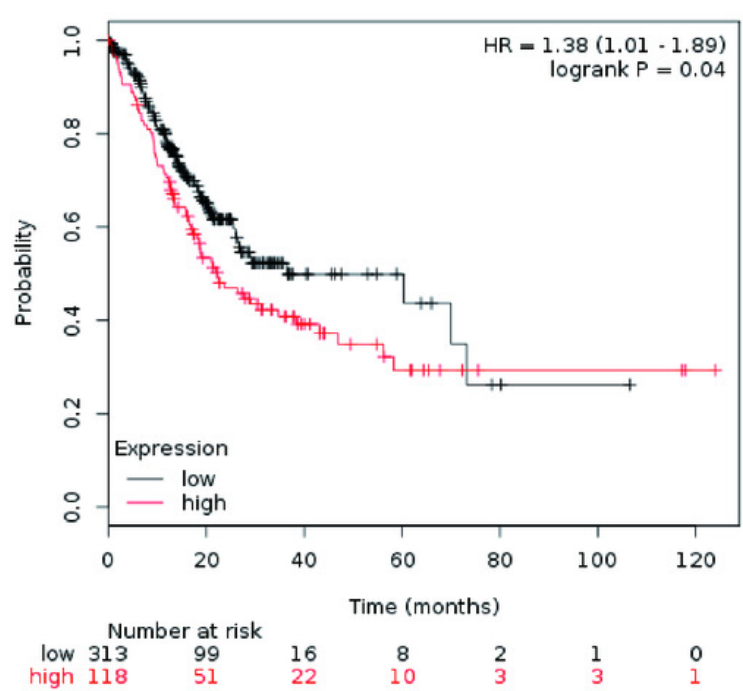

$\mathbf{F}$

hsa-mir-433

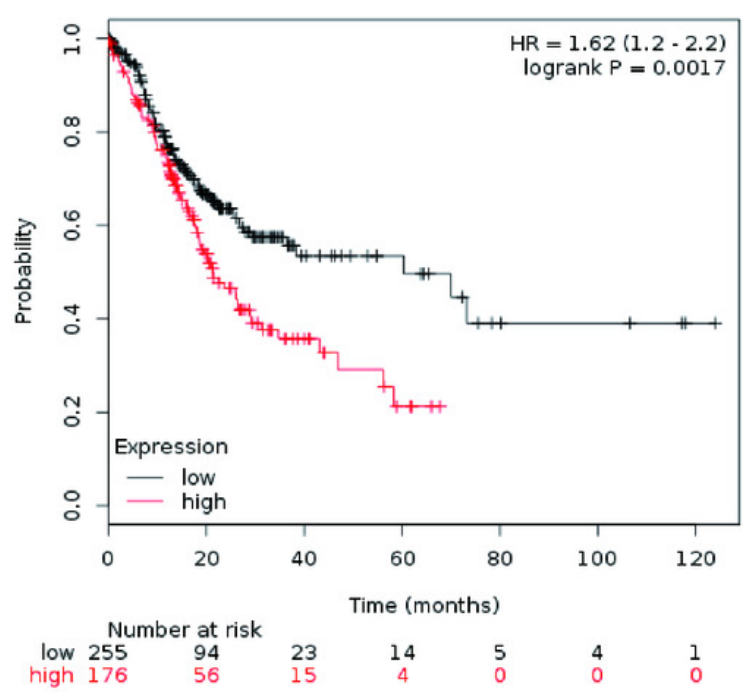


Figure 3

Bioinformatics analysis of selected miRNAs.

(A-G) Based on TargetScan, miRDB, miRTarBase and miRWalk, the predict targets gene of miR-9-3p (A), miR-9-5p (B), miR-146a-5p (C), miR-370-3p (D), miR-433-3p (E), miR-519a-5p

(F) and miR-522-5p (G) were selected using Venn graphing. 

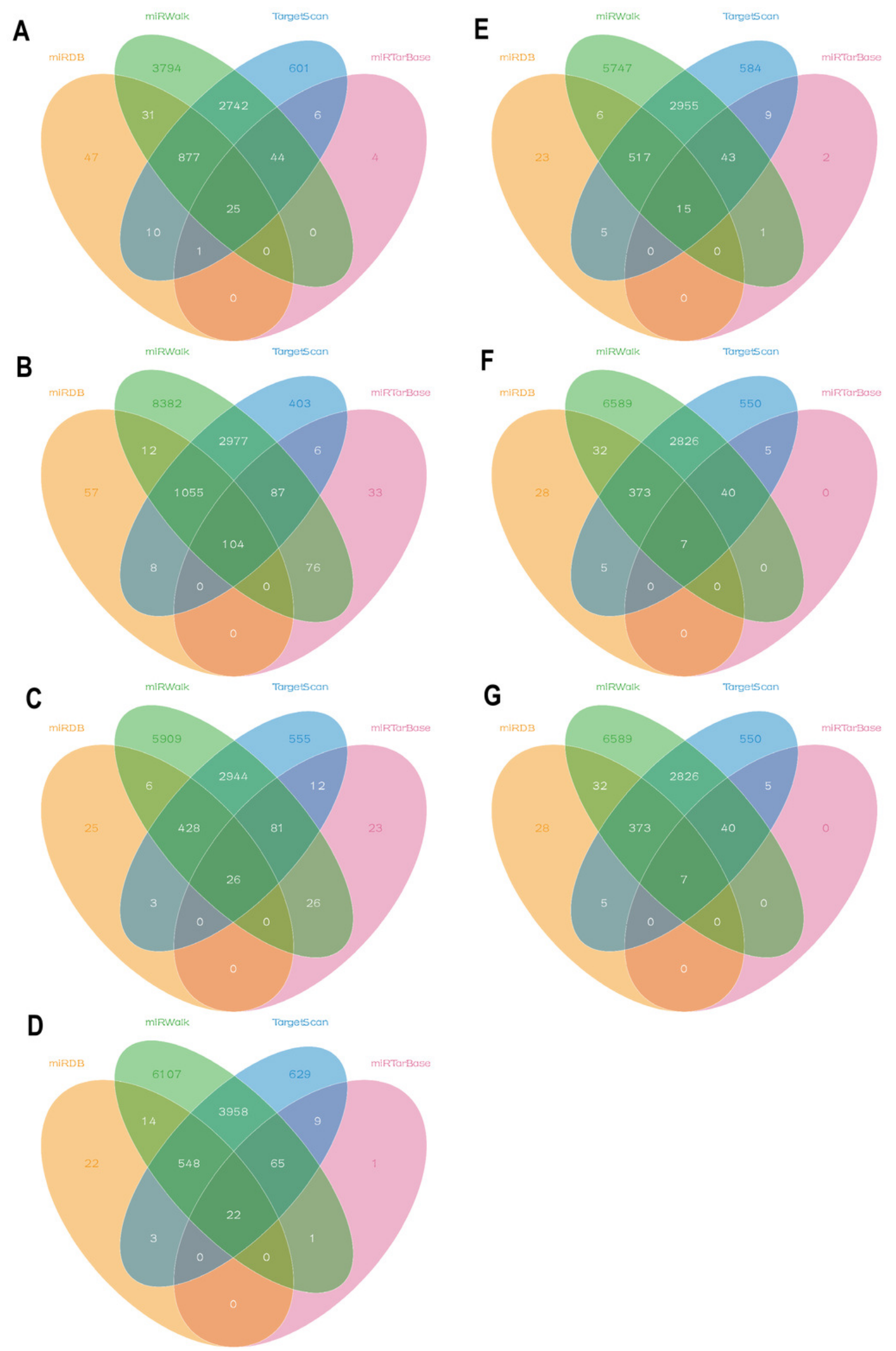


\section{Figure 4}

Expression levels and functions of 7 selected miRNAs in human GC cells and clinical specimens.

(A) The relative level (fold change) of these seven selected miRNAs between DDP-resistant MGC803/DDP cells and parental MGC803 cells was analyzed via RT-qPCR. (B-E) The relative levels of miR-9-3p (B), miR-9-5p (C), miR-146a-5p (D), and miR-433-3p (E) between 34 chemotherapy response sensitive gastric cancer serums and 40 chemotherapy response resistant gastric cancer serums were significantly different, which were measured using RTqPCR. (F-H) The relative levels of miR-370-3p (F), miR-519a-5p (G), and miR-522-5p (H) between 34 chemotherapy response sensitive gastric cancer serums and 40 chemotherapy response resistant gastric cancer serums didn't show significantly different, which were measured using RT-qPCR. Each assay was conducted in triplicate. ${ }^{* * * * *} \mathrm{P}<0.0001,{ }^{* * *} \mathrm{P}<0.001$, ${ }^{* *} \mathrm{P}<0.01,{ }^{*} \mathrm{P}<0.05$ and mean \pm SD were utilized to show the data. 

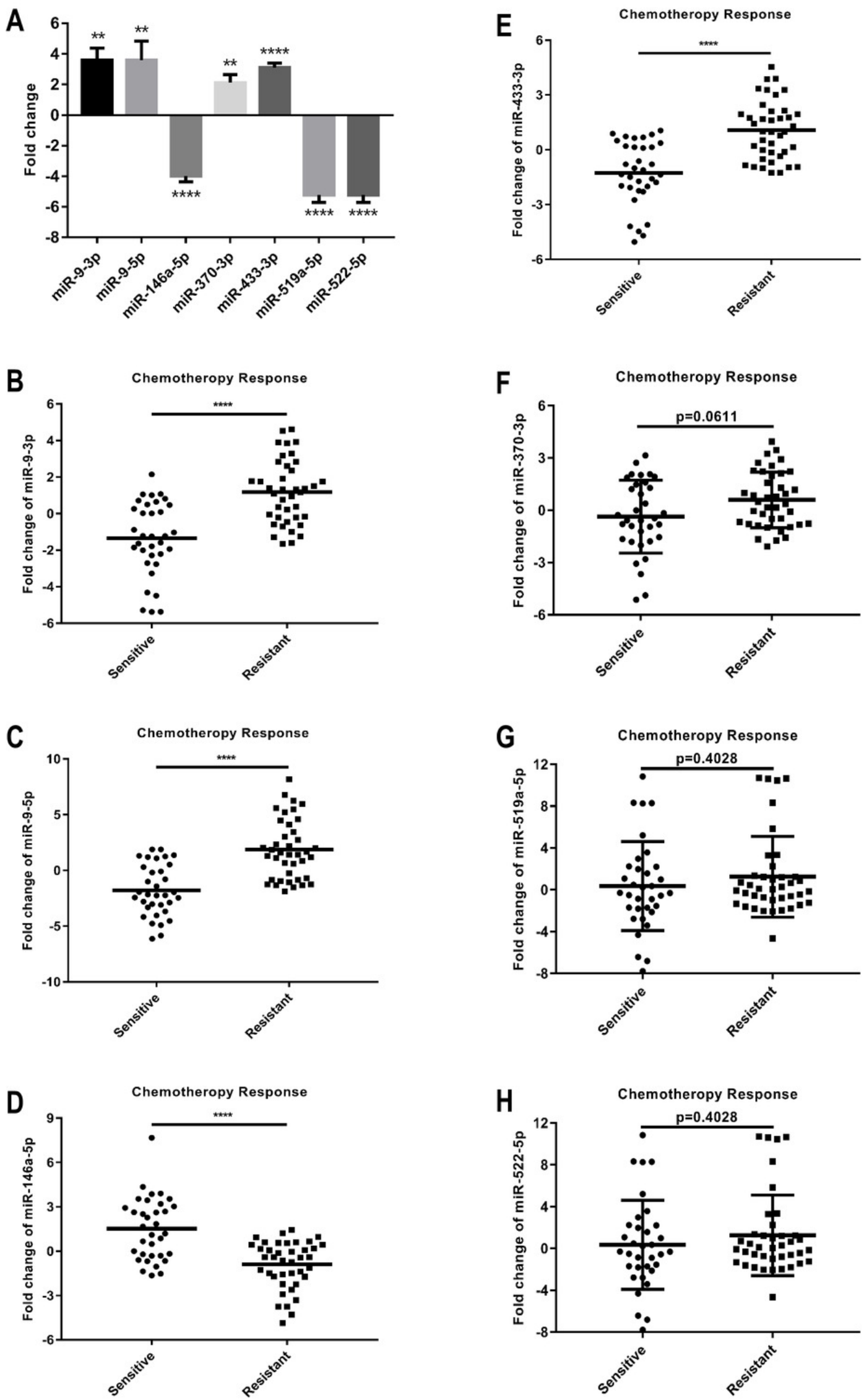
Figure 5

ROC curve and AUC value in comparison of the prognostic accuracy for DDP response with single miRNA expression.

(A-D)ROC curves and AUC values of miR-9-3p (A), miR-9-5p (B), miR-146a-5p (C) and miR-433-3p (D) distinguished the GC chemotherapy response-resistant group from the GC chemotherapy response-sensitive group.

A

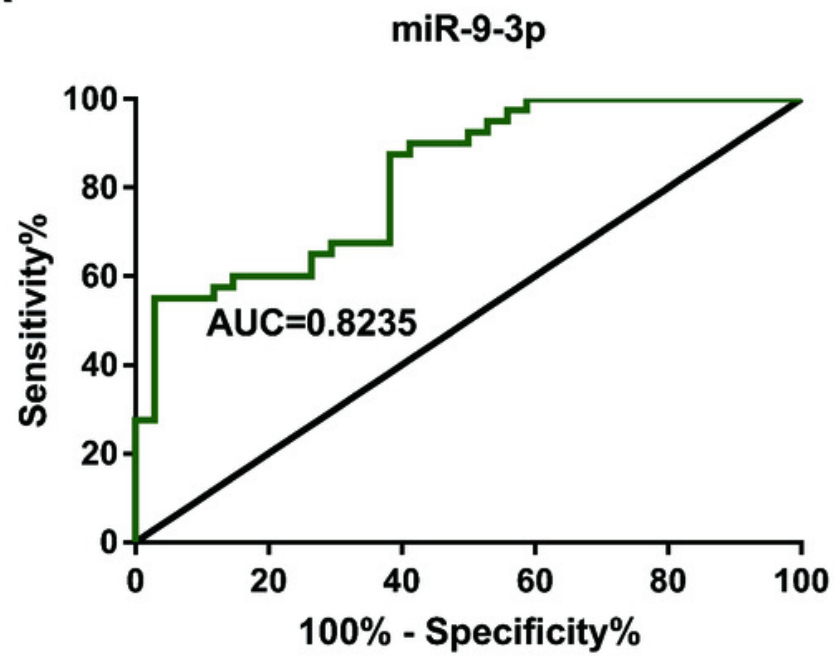

C

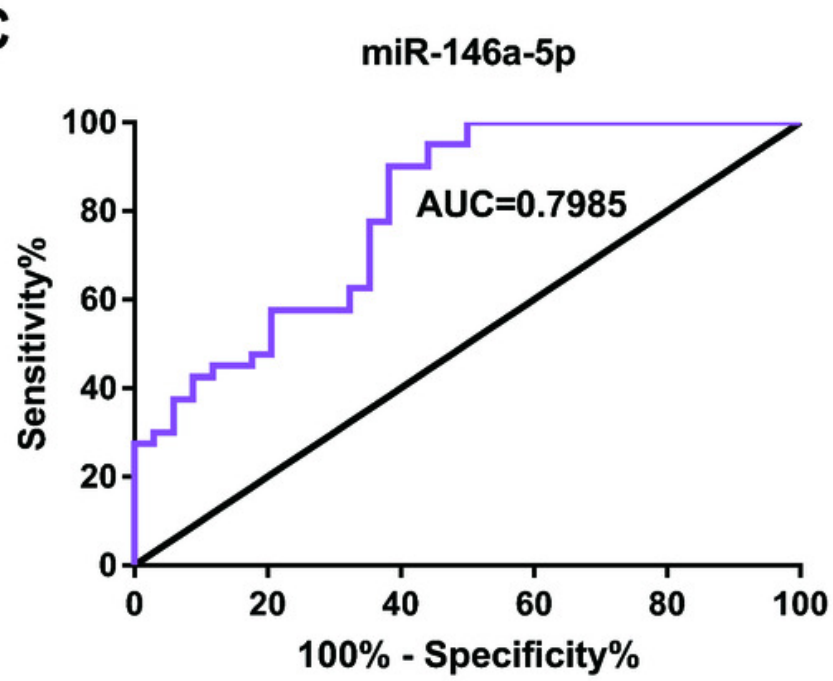

B

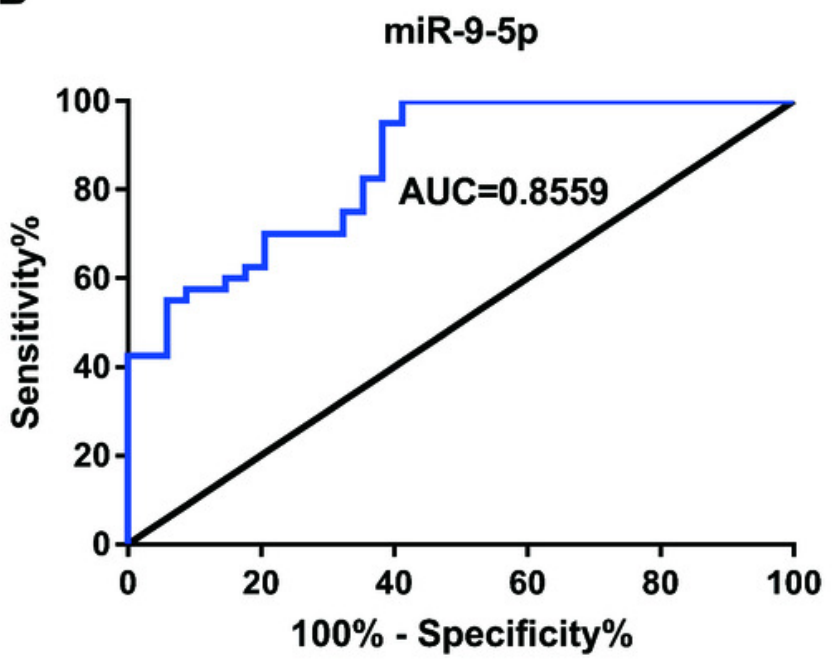

D

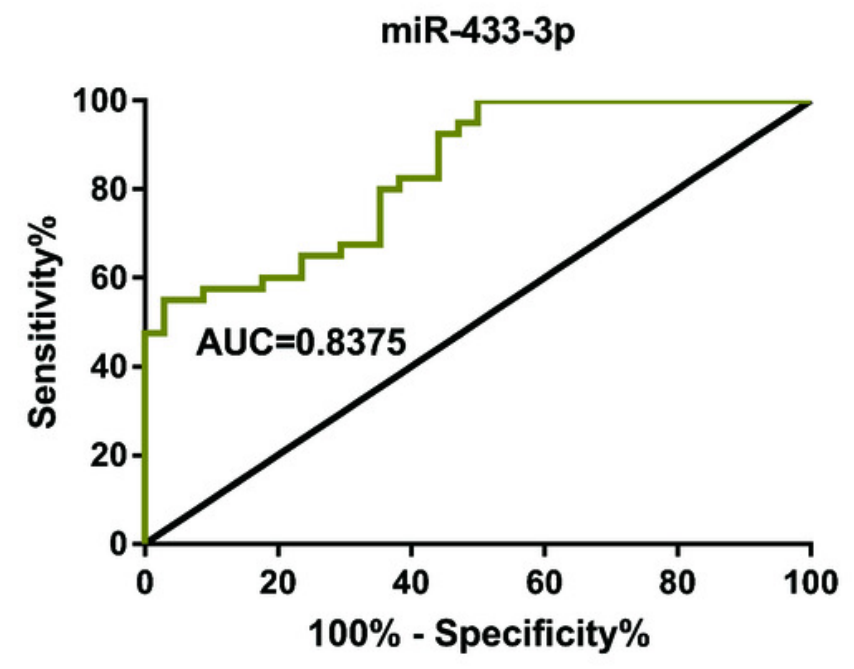




\section{Figure 6}

ROC curve and AUC value in comparison of the prognostic accuracy for DDP response with two combined miRNAs expression.

(A-F)ROC curves and AUC values of miR-9-3p combined with miR-9-5p (A), miR-9-3p combined with miR-146a-5p (B), miR-9-3p combined with miR-433-3p (C) , miR-9-5p combined with miR-146a-5p (D), miR-9-5p combined with miR-433-3p (E) and miR-146a-5p combined with miR-433-3p (F) distinguished the GC chemotherapy response-resistant group from the GC chemotherapy response-sensitive group. 
A

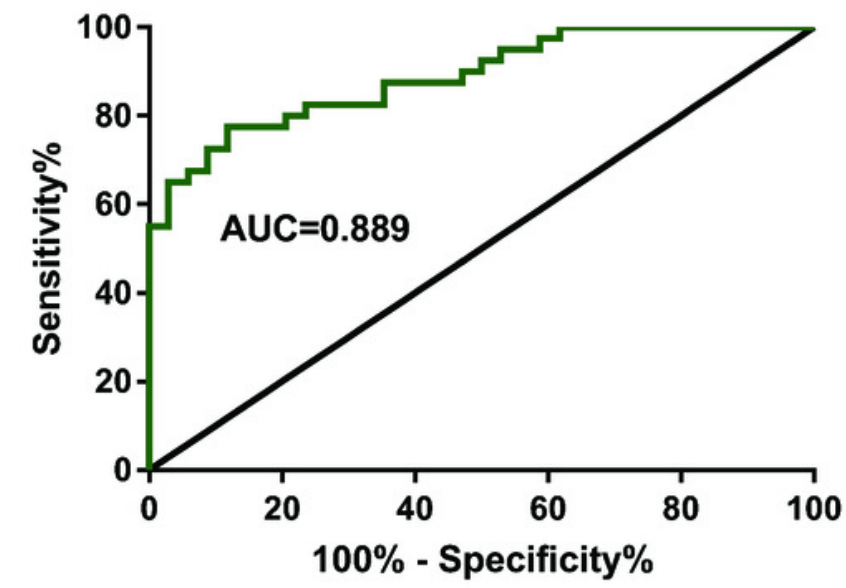

$\boldsymbol{B}$

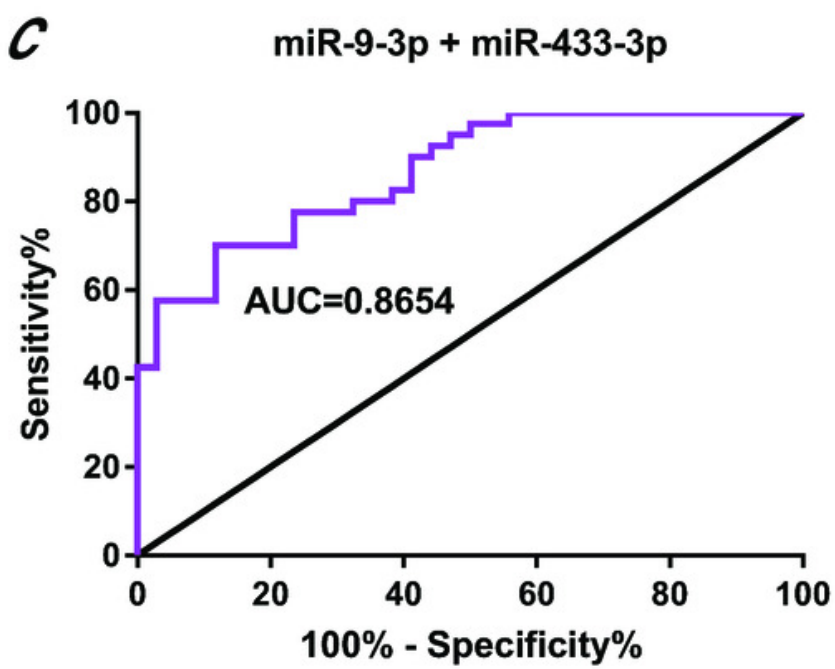

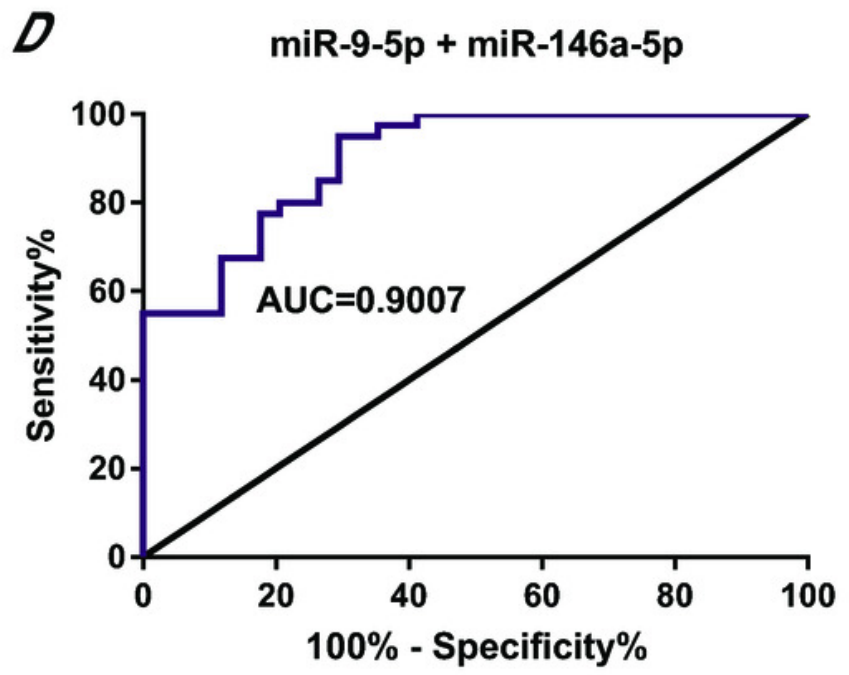
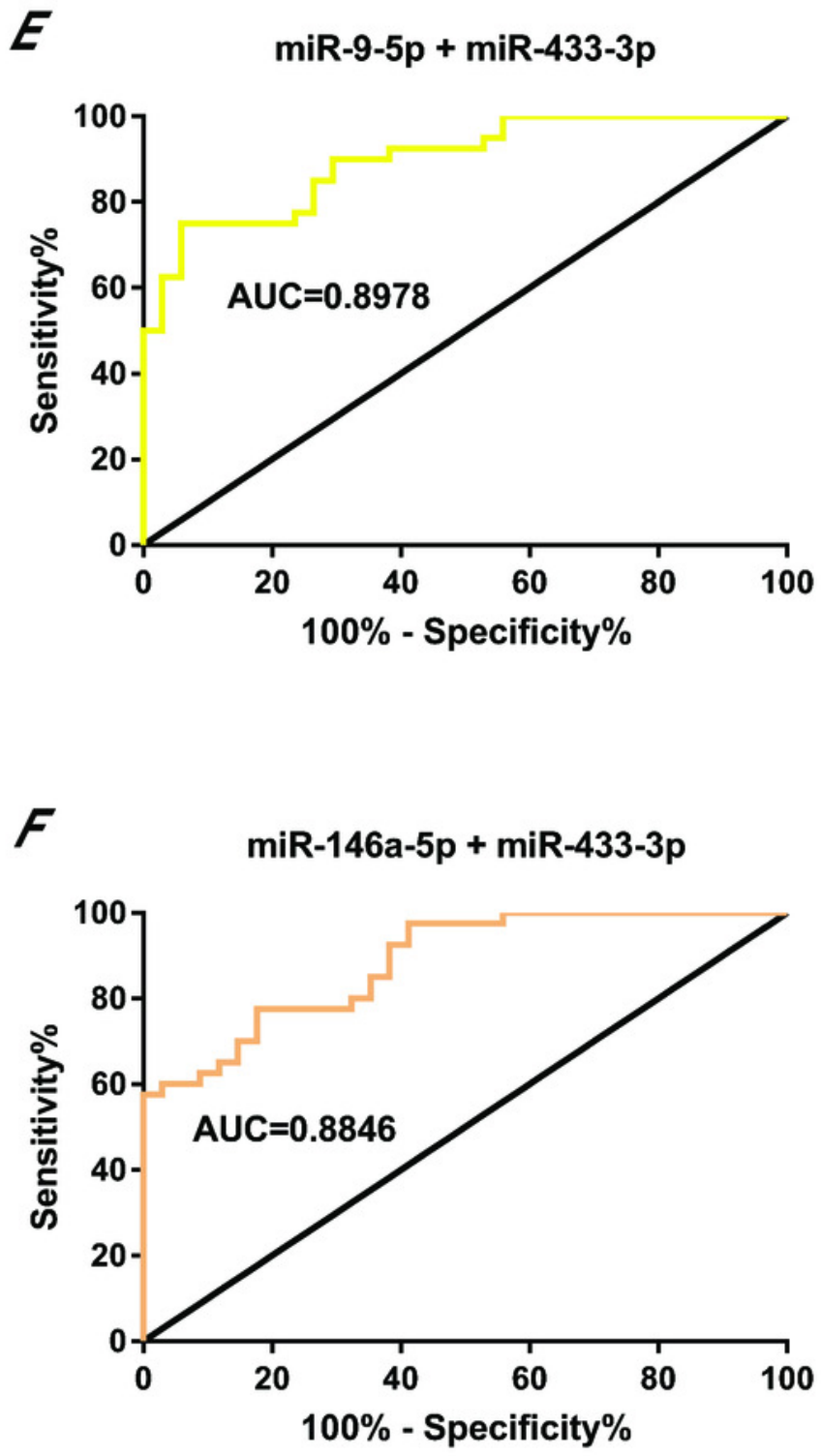


\section{Figure 7}

ROC curve and AUC value in comparison of the prognostic accuracy for DDP response with three or more combined miRNAs expression.

(A-E)ROC curves and AUC values of miR-9-3p combined with miR-9-5p and miR-146a-5p (A), miR-9-3p combined with miR-9-5p and miR-433-3p (B), miR-9-3p combined with miR-146a-5p and miR-433-3p (C) , miR-9-5p combined with miR-146a-5p and miR-433-3p (D), and miR-9-3p combined with miR-9-5p, miR-146a-5p and miR-433-3p (E) distinguished the GC chemotherapy response-resistant group from the GC chemotherapy responsesensitive group. 
A

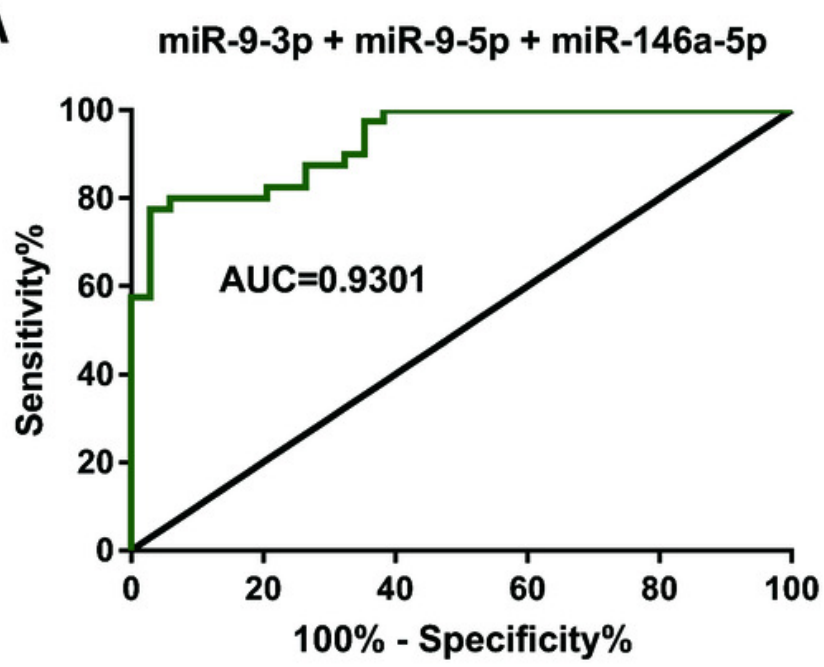

C

$m i R-9-3 p+m i R-146 a-5 p+m i R-433-3 p$

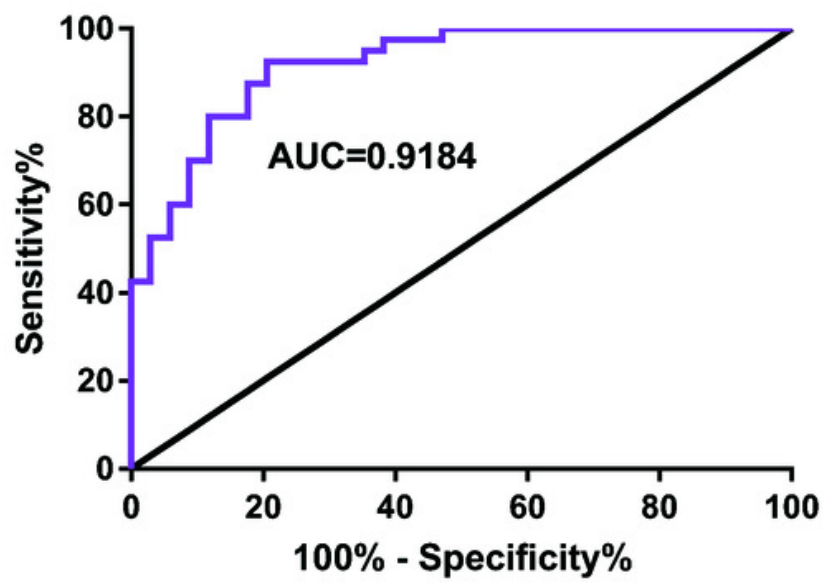

E $m i R-9-3 p+m i R-9-5 p+m i R-146 a-5 p+m i R-433-3 p$

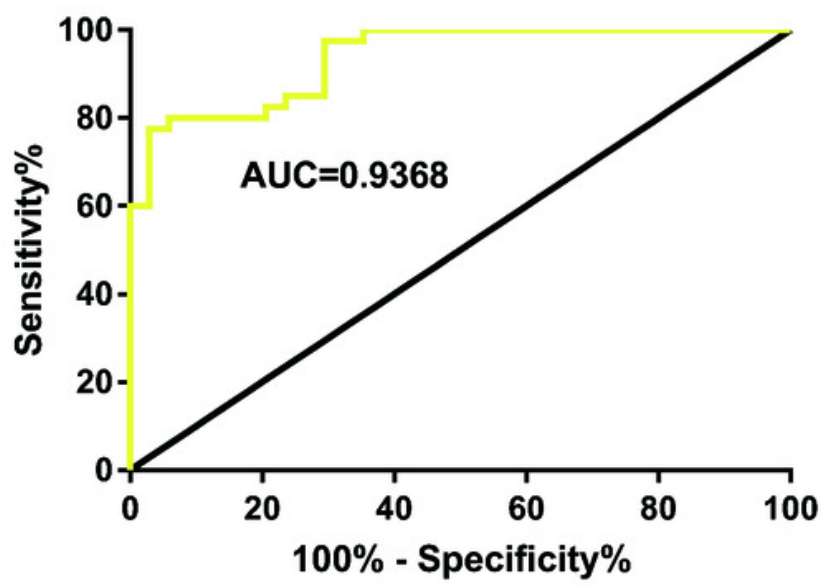

PeerJ reviewing PDF | (2019:10:41928:1:2:NEW 18 Feb 2020)
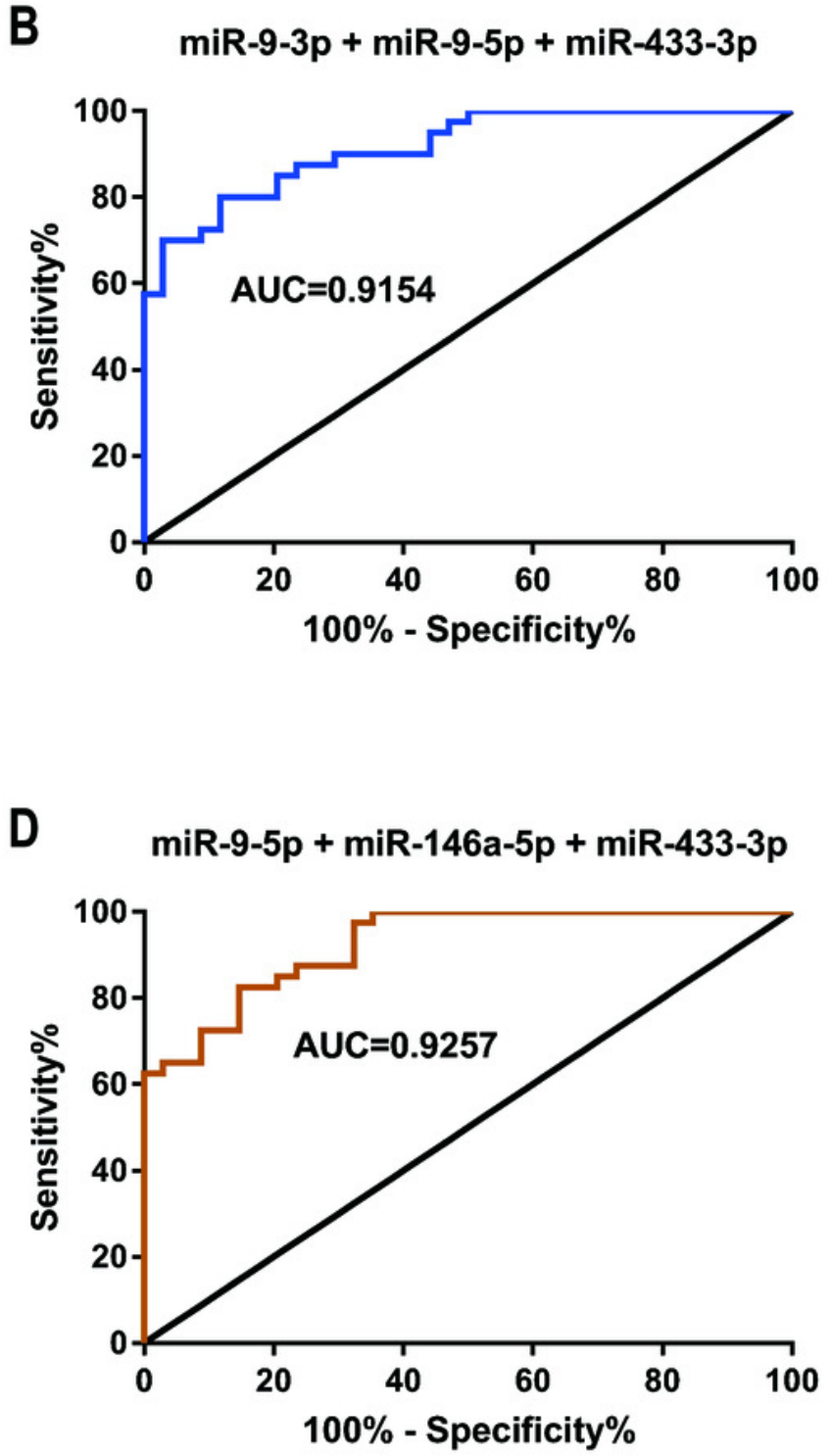


\section{Figure 8}

PairwisecomparisonofROCcurves by the MedCalc software.

(A)The two compared areas between the miR-9-5p + miR-9-3p + miR-146a-5p group and the miR-9-5p group were significantly different. (B)The two compared areas between the $m i R-9-5 p+m i R-9-3 p+m i R-433-3 p$ group and the miR-9-5p group were significantly different. (C)The two compared areas between the miR-9-5p + miR-146a-5p + miR-433-3p group and the miR-9-5p group were significantly different. (D) The two compared areas between the miR-9-5p + miR-9-3p + miR-146a-5p + miR-433-3p group and the miR-9-5p group were significantly different. ${ }^{* * * * *} \mathrm{P}<0.0001,{ }^{* * * *} \mathrm{P}<0.001,{ }^{* *} \mathrm{P}<0.01,{ }^{*} \mathrm{P}<0.05$ and mean \pm SD were utilized to show the data. 

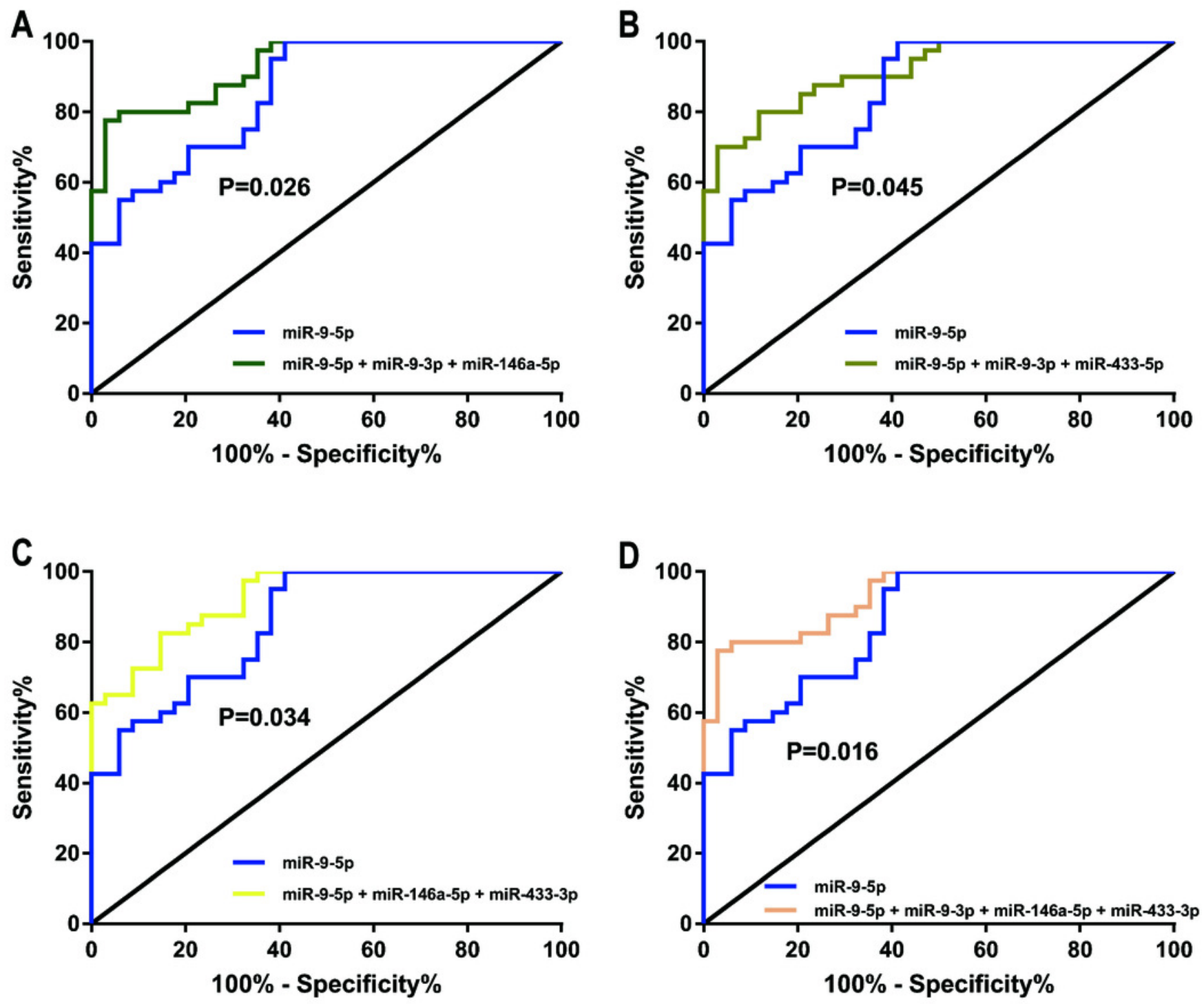


\section{Figure 9}

Three selected miRNAs were ascertained in two public databases for prognostic analysis.

(A, B) Kaplan-Meier survival curves suggested that patients with high miR-9-3p levels had shorter OS times than those with low miR-9-3p levels in the OncoLnc database ( $\mathbf{A}$, $P=0.00836$ ) and in the OncomiR database (B, $P=0.06643)$. (C, D) Kaplan-Meier survival curves suggested that patients with high miR-9-5p levels had shorter OS times than those with low miR-9-5p levels in the OncoLnc database (C, $P=0.00181)$ and in the OncomiR database ( $\mathbf{D}, \mathbf{P}=0.0044)$. (E, F) Kaplan-Meier survival curves suggested that patients with high miR-433-3p levels had shorter OS times than those with low miR-433-3p levels in the Oncolnc database $(\mathbf{E}, \mathrm{P}=0.0311)$ and in the OncomiR database $(\mathbf{F}, \mathrm{P}=0.05651) .{ }^{* * * *} \mathrm{P}<$ $0.0001,{ }^{* * *} \mathrm{P}<0.001,{ }^{* * *} \mathrm{P}<0.01,{ }^{*} \mathrm{P}<0.05$ and mean \pm SD were utilized to show the data. 
A

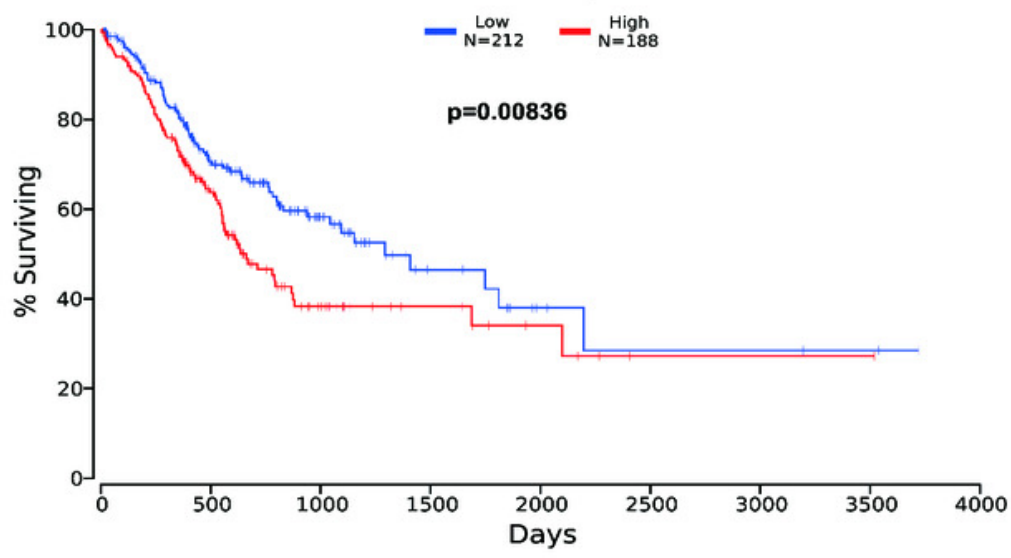

C

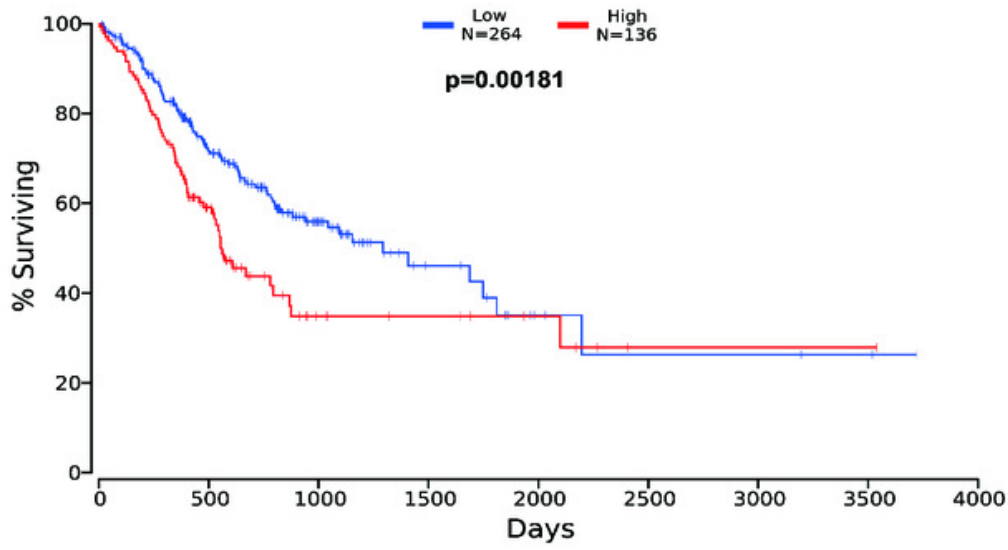

E

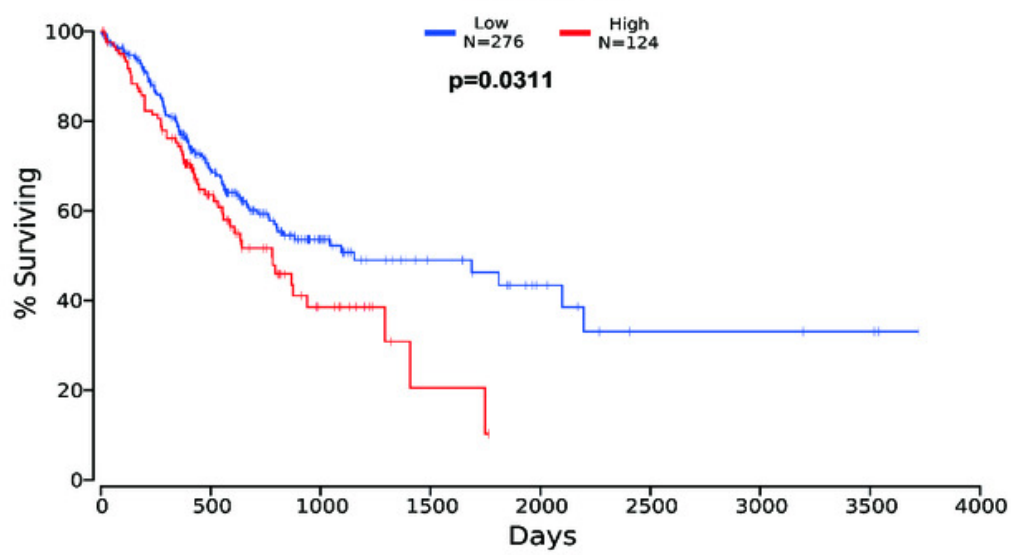

B

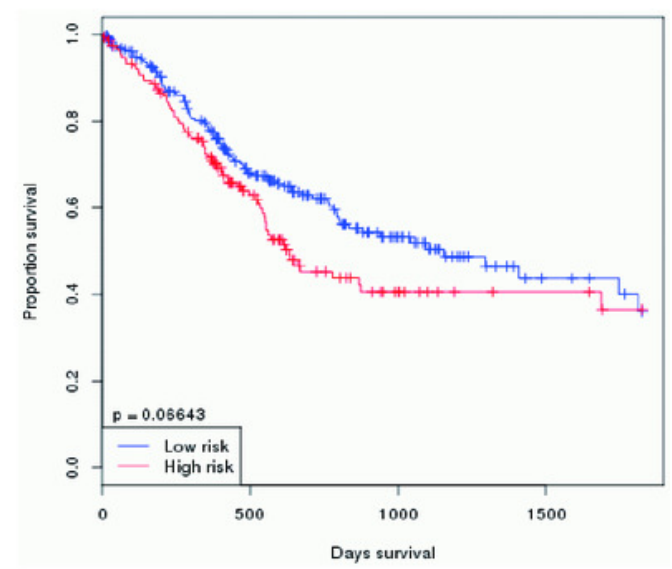

D

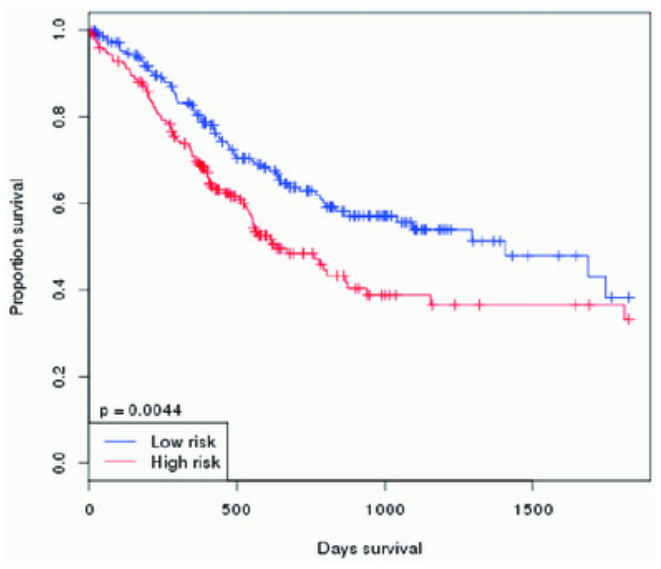

F

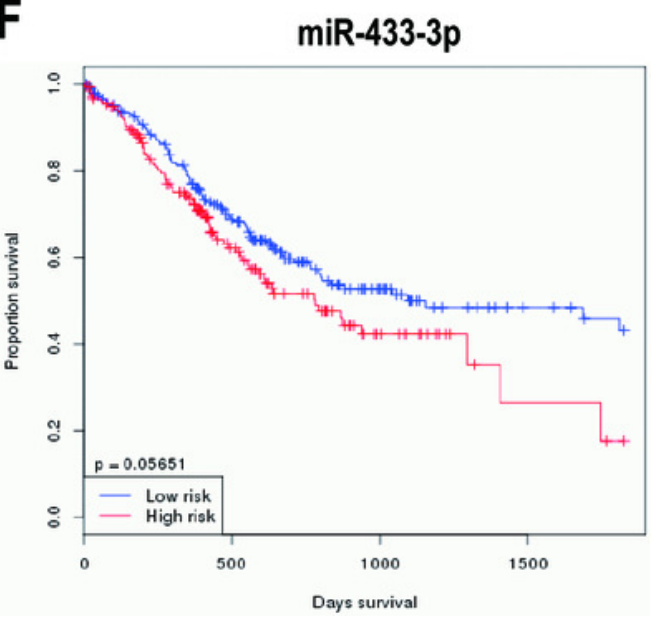




\section{Figure 10}

Different miRNA combinations were ascertained in two public databases for prognostic analysis.

(A)Kaplan-Meier survival curves suggested that patients with high miR-9-3p + miR-9-5p levels had shorter OS times than those with low miR-9-3p + miR-9-5p levels in the OncomiR database ( $P=0.003421)$. (B) Kaplan-Meier survival curves suggested that patients with high miR-9-5p + miR-433-3p levels had shorter OS times than those with low miR-9-5p + miR-433-3p levels in the OncomiR database $(P=0.01428)$. (C) Kaplan-Meier survival curves suggested that patients with high miR-9-3p + miR-9-5p + miR-433-3p levels had shorter OS times than those with low miR-9-3p + miR-9-5p + miR-433-3p levels in the OncomiR database $(P=0.008066)$. (D) Kaplan-Meier survival curves suggested that patients with high miR-9 + miR-433 levels had shorter OS times than those with low miR-9 + miR-433 levels in the Kaplan-Meier Plotter database $(P=0.0047) .{ }^{* * * *} P<0.0001,{ }^{* * *} \mathrm{P}<0.001,{ }^{* *} \mathrm{P}<0.01,{ }^{*} \mathrm{P}<$ 0.05 and mean \pm SD were utilized to show the data. 
A

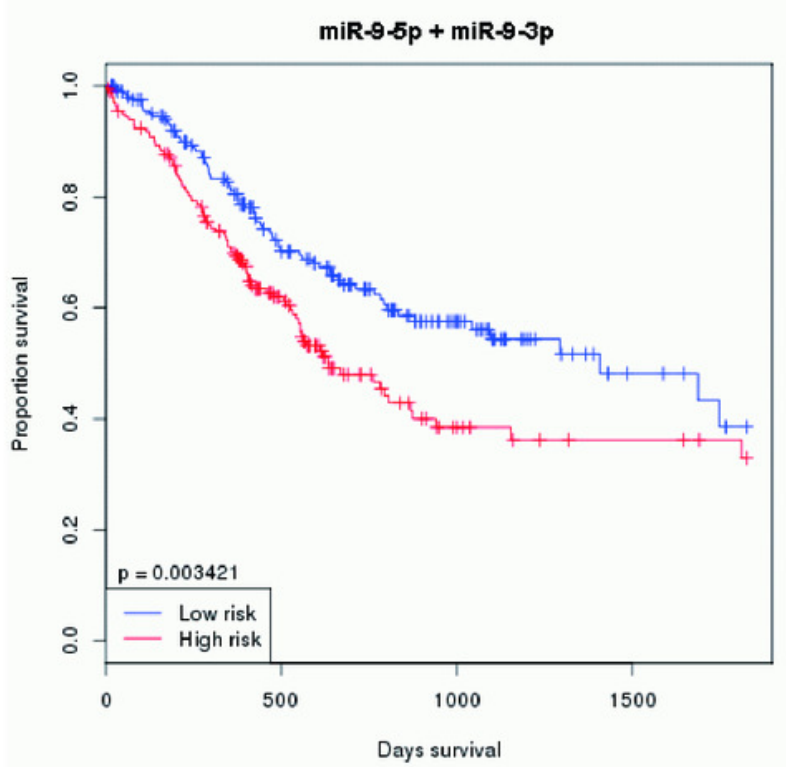

C

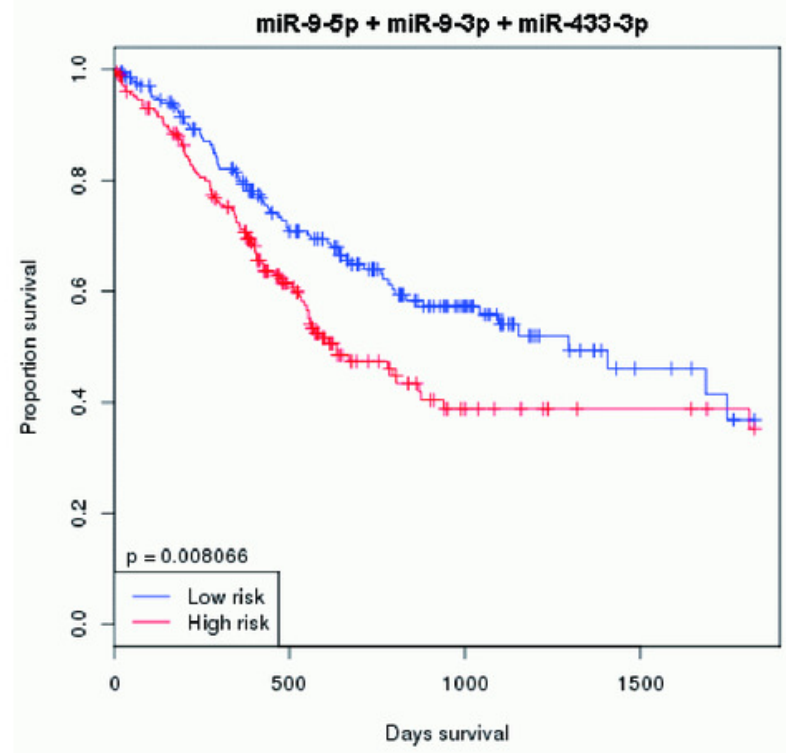

B

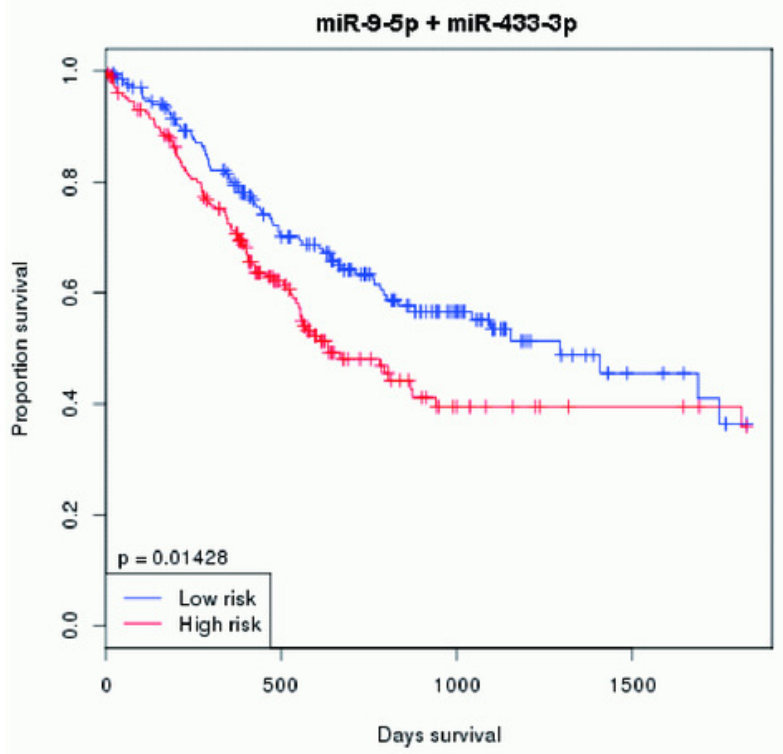

D

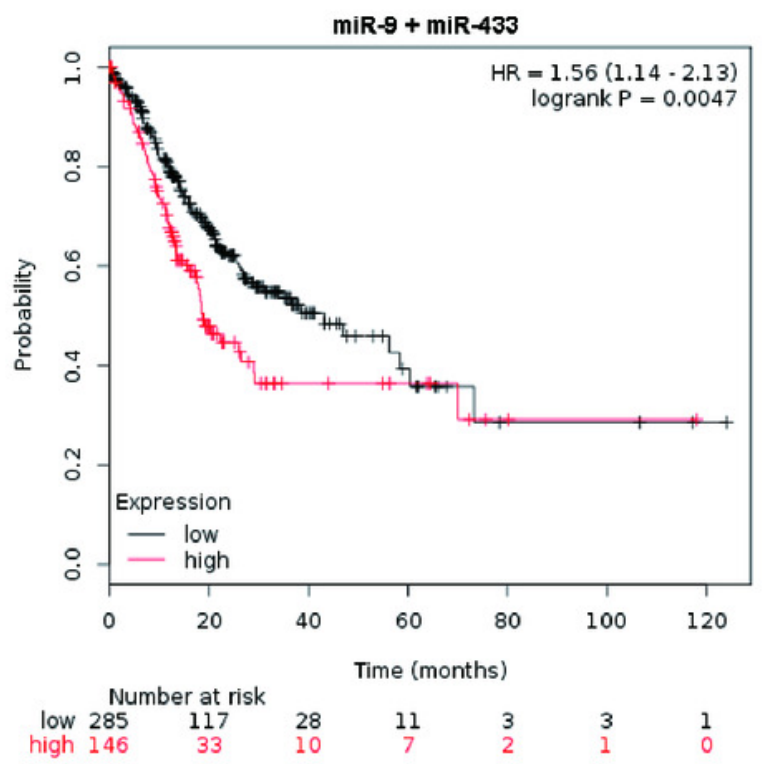


Table $\mathbf{1}$ (on next page)

Primers of RT-qPCR. 
Primers of RT-qPCR.

\begin{tabular}{lll}
\hline miRNA symbol & Article number & Sequence \\
\hline miR-9-3p & HmiRQP0824 & CCATAAAGCTAGATAACCGAAAGTAA \\
miR-9-5p & HmiRQP0825 & TCTTTGGTTATCTAGCTGTATGAAA \\
miR-146a-5p & HmiRQP0196 & AGAACTGAATTCCATGGGTTAA \\
miR-370-3p & HmiRQP0456 & GCTGGGGTGGAACCTGGTAA \\
miR-433-3p & HmiRQP0502 & TGATGGGCTCCTCGGTGTAA \\
miR-519a-5p & HmiRQP0591 & TAGAGGGAAGCGCTTTCTGAAA \\
miR-522-5p & HmiRQP0591 & TAGAGGGAAGCGCTTTCTGAAA \\
let-7g-5p & HmiRQP0015 & TGAGGTAGTAGTTTGTACAGTTAA \\
U6 & HmiRQP9001 & Not available \\
\hline
\end{tabular}

1 


\section{Table 2 (on next page)}

35 differentially expressed miRNAs in sRNA-seq data and Kaplan-Meier Plotter analysis. 
35 differentially expressed miRNAs in sRNA-seq data and Kaplan-Meier Plotter analysis.

\begin{tabular}{|c|c|c|c|c|c|c|}
\hline \multirow{2}{*}{ miRNA } & \multicolumn{2}{|c|}{ Counts } & \multirow{2}{*}{$\log _{2} \mathrm{FC}$} & \multirow{2}{*}{ P-value } & \multicolumn{2}{|c|}{ Kaplan-Meier Plotter } \\
\hline & MGC803 & MGC803/DDP & & & Hazard Ratio & P-value \\
\hline hsa-miR-124-3p & 457 & 0 & -12.2235 & $1.2576 \mathrm{E}-16$ & $0.75(0.55 \sim 1.02)$ & 0.0610 \\
\hline hsa-miR-9-5p & 1155 & 12 & -7.2625 & $1.0889 \mathrm{E}-15$ & $1.56(1.14 \sim 2.13)$ & $0.0047^{* *}$ \\
\hline hsa-miR-204-5p & 238 & 3 & -6.9301 & $4.0321 \mathrm{E}-12$ & $0.73(0.50 \sim 1.06)$ & 0.0930 \\
\hline hsa-miR-146a-5p & 4 & 486 & 6.1991 & $4.1414 \mathrm{E}-11$ & $0.70(0.52 \sim 0.95)$ & $0.0230^{*}$ \\
\hline hsa-miR-129-5p & 352 & 14 & -5.3287 & $1.9485 \mathrm{E}-10$ & $1.39(0.99 \sim 1.93)$ & 0.0540 \\
\hline hsa-miR-127-3p & 84 & 1 & -6.8789 & $1.1322 \mathrm{E}-09$ & $1.30(0.95 \sim 1.79)$ & 0.0990 \\
\hline hsa-miR-211-5p & 62 & 0 & -9.3436 & $1.4691 \mathrm{E}-08$ & $0.74(0.53 \sim 1.04)$ & 0.0810 \\
\hline hsa-miR-486-5p & 21 & 298 & 3.1290 & $3.7724 \mathrm{E}-05$ & $0.77(0.55 \sim 1.07)$ & 0.1200 \\
\hline hsa-miR-9-3p & 18 & 0 & -7.5647 & 0.0002 & $1.56(1.14 \sim 2.13)$ & $0.0047^{* *}$ \\
\hline hsa-miR-381-3p & 13 & 0 & -7.0982 & 0.0010 & $1.26(0.90 \sim 1.75)$ & 0.1700 \\
\hline hsa-miR-3180 & 0 & 20 & 7.0281 & 0.0010 & $0.76(0.55 \sim 1.05)$ & 0.0910 \\
\hline hsa-miR-3180-3p & 0 & 20 & 7.0281 & 0.0010 & $0.76(0.55 \sim 1.05)$ & 0.0910 \\
\hline hsa-miR-370-3p & 11 & 0 & -6.8591 & 0.0029 & $1.56(1.14 \sim 2.13)$ & $0.0053^{* *}$ \\
\hline hsa-miR-135a-5p & 10 & 0 & -6.7228 & 0.0042 & $1.16(0.85 \sim 1.58)$ & 0.3500 \\
\hline hsa-miR-146b-5p & 78 & 481 & 1.9311 & 0.0058 & $1.16(0.83 \sim 1.62)$ & 0.3900 \\
\hline hsa-miR-522-5p & 9 & 0 & -6.5723 & 0.0061 & $1.38(1.01 \sim 1.89)$ & $0.0400^{*}$ \\
\hline hsa-miR-519b-5p & 9 & 0 & -6.5723 & 0.0061 & $0.79(0.58 \sim 1.07)$ & 0.1200 \\
\hline hsa-miR-519a-5p & 9 & 0 & -6.5723 & 0.0061 & $1.39(1.02 \sim 1.91)$ & $0.0360^{*}$ \\
\hline hsa-miR-518e-5p & 9 & 0 & -6.5723 & 0.0061 & $0.78(0.57 \sim 1.06)$ & 0.1200 \\
\hline hsa-miR-523-5p & 9 & 0 & -6.5723 & 0.0061 & $0.77(0.57 \sim 1.05)$ & 0.0930 \\
\hline hsa-miR-519c-5p & 9 & 0 & -6.5723 & 0.0061 & $0.79(0.58 \sim 1.08)$ & 0.1400 \\
\hline hsa-miR-7977 & 0 & 14 & 6.5182 & 0.0061 & $0.75(0.54 \sim 1.05)$ & 0.0900 \\
\hline hsa-miR-543 & 8 & 0 & -6.4043 & 0.0092 & $0.76(0.55 \sim 1.05)$ & 0.0990 \\
\hline hsa-miR-433-3p & 8 & 0 & -6.4043 & 0.0092 & $1.62(1.20 \sim 2.20)$ & $0.0017^{* *}$ \\
\hline hsa-miR-1244 & 16 & 4 & -2.6460 & 0.0144 & $0.74(0.53 \sim 1.02)$ & 0.0630 \\
\hline hsa-miR-486-3p & 0 & 10 & 6.0391 & 0.0219 & $0.77(0.55 \sim 1.07)$ & 0.1200 \\
\hline hsa-miR-6724-5p & 28 & 12 & -1.9009 & 0.0264 & $0.74(0.53 \sim 1.02)$ & 0.0630 \\
\hline hsa-miR-663a & 32 & 14 & -1.8732 & 0.0287 & $0.74(0.53 \sim 1.02)$ & 0.0630 \\
\hline hsa-miR-124-5p & 6 & 0 & -5.9949 & 0.0348 & $0.75(0.55 \sim 1.02)$ & 0.0610 \\
\hline hsa-miR-382-5p & 6 & 0 & -5.9949 & 0.0348 & $1.31(0.95 \sim 1.81)$ & 0.1000 \\
\hline hsa-miR-1306-3p & 0 & 9 & 5.8895 & 0.0348 & $0.82(0.60 \sim 1.13)$ & 0.2300 \\
\hline hsa-miR-1-3p & 0 & 8 & 5.7226 & 0.0348 & $1.30(0.95 \sim 1.76)$ & 0.0980 \\
\hline hsa-miR-1285-5p & 30 & 15 & -1.6819 & 0.0366 & $0.74(0.53 \sim 1.02)$ & 0.0630 \\
\hline hsa-miR-3913-5p & 2 & 20 & 2.5736 & 0.0382 & $1.23(0.88 \sim 1.72)$ & 0.2300 \\
\hline hsa-miR-181a-2-3p & 60 & 260 & 1.4220 & 0.0429 & $1.35(0.99 \sim 1.84)$ & 0.0530 \\
\hline
\end{tabular}




\section{Table 3 (on next page)}

7 selected miRNAs in SRNA-seq data and RT-qPCR verification in GC cells. 
7 selected miRNAs in sRNA-seq data and RT-qPCR verification in GC cells.

\begin{tabular}{lcccc}
\hline \multirow{2}{*}{ miRNA } & \multicolumn{2}{c}{ sRNA-seq } & \multicolumn{2}{c}{ RT-qPCR } \\
\cline { 2 - 5 } & $\log _{2}$ FC & P-value & $\log _{2}$ FC & P-value \\
\hline hsa-miR-9-3p & -7.5647 & 0.0002 & 3.6005 & $0.0013^{* * *}$ \\
hsa-miR-9-5p & -7.2625 & $1.0889 \mathrm{E}-15$ & 3.5865 & $0.0076^{* *}$ \\
hsa-miR-146a-5p & 6.1991 & $4.1414 \mathrm{E}-11$ & -3.9956 & $<0.0001^{* * * *}$ \\
hsa-miR-370-3p & -6.8591 & 0.0029 & 2.1172 & $0.0022^{* *}$ \\
hsa-miR-433-3p & -6.4043 & 0.0092 & 3.1024 & $<0.0001^{* * * *}$ \\
hsa-miR-519a-5p & -6.5723 & 0.0061 & -5.2120 & $<0.0001^{* * * *}$ \\
hsa-miR-522-5p & -6.5723 & 0.0061 & -5.2120 & $<0.0001^{* * * *}$ \\
\hline
\end{tabular}




\section{Table 4 (on next page)}

The correlation between miRNA expression and clinical parameters in GC patients $(n=74)$. 
The correlation between miRNA expression and clinical parameters in GC patients ( $n=74)$.

\begin{tabular}{|c|c|c|c|c|c|c|c|c|c|c|}
\hline \multirow{2}{*}{ miRNA level } & \multirow{2}{*}{ Cases(n) } & \multicolumn{3}{|c|}{ Gender } & \multicolumn{3}{|c|}{ Age (years) } & \multicolumn{3}{|c|}{ Chemotherapy response } \\
\hline & & Male & Female & P-value & $<60$ & $\geqslant 60$ & P-value & Sensitive & Resistant & P-value \\
\hline miR-9-3p & 74 & 52 & 22 & & 23 & 51 & & 34 & 40 & \\
\hline low & 37 & 24 & 13 & 0.2413 & 9 & 28 & 0.5856 & 24 & 13 & $<0.0001^{* * * * *}$ \\
\hline high & 37 & 28 & 9 & & 14 & 23 & & 10 & 27 & \\
\hline miR-9-5p & 74 & 52 & 22 & & 23 & 51 & & 34 & 40 & \\
\hline low & 37 & 28 & 9 & 0.8557 & 11 & 26 & 0.9262 & 26 & 11 & $<0.0001^{* * * *}$ \\
\hline high & 37 & 24 & 13 & & 12 & 25 & & 8 & 29 & \\
\hline miR-146a-5p & 74 & 52 & 22 & & 23 & 51 & & 34 & 40 & \\
\hline low & 37 & 26 & 11 & 0.7469 & 12 & 25 & 0.6510 & 12 & 25 & $<0.0001^{* * * *}$ \\
\hline high & 37 & 26 & 11 & & 11 & 26 & & 22 & 15 & \\
\hline miR-370-3p & 74 & 52 & 22 & & 23 & 51 & & 34 & 40 & \\
\hline low & 37 & 23 & 14 & 0.1757 & 13 & 24 & 0.8257 & 20 & 17 & 0.0611 \\
\hline high & 37 & 29 & 8 & & 10 & 27 & & 14 & 23 & \\
\hline miR-433-3p & 74 & 52 & 22 & & 23 & 51 & & 34 & 40 & \\
\hline low & 37 & 24 & 13 & 0.1848 & 9 & 28 & 0.3408 & 24 & 13 & $<0.0001^{* * * * *}$ \\
\hline high & 37 & 28 & 9 & & 14 & 23 & & 10 & 27 & \\
\hline miR-519a-5p & 74 & 52 & 22 & & 23 & 51 & & 34 & 40 & \\
\hline low & 37 & 24 & 13 & 0.4414 & 9 & 28 & 0.1552 & 18 & 19 & 0.4028 \\
\hline high & 37 & 28 & 9 & & 14 & 23 & & 16 & 21 & \\
\hline miR-522-5p & 74 & 52 & 22 & & 23 & 51 & & 34 & 40 & \\
\hline low & 37 & 24 & 13 & 0.4414 & 9 & 28 & 0.1552 & 18 & 19 & 0.4028 \\
\hline high & 37 & 28 & 9 & & 14 & 23 & & 16 & 21 & \\
\hline
\end{tabular}


Table 5 (on next page)

ROC analysis of miRNAs. 
ROC analysis of miRNAs.

\begin{tabular}{|c|c|c|c|c|c|c|}
\hline miRNA & $A \cup C$ & SD & $95 \% \mathrm{Cl}$ & $\begin{array}{l}\text { SE } \\
(\%)\end{array}$ & $\begin{array}{l}\mathrm{SP} \\
(\%)\end{array}$ & $P$ value \\
\hline miR-9-3p & $\begin{array}{c}0.82 \\
4\end{array}$ & $\begin{array}{c}0.04 \\
7\end{array}$ & $0.731-0.916$ & 70.6 & 67.5 & $<0.0001^{* * * *}$ \\
\hline $\operatorname{miR}-9-5 p$ & $\begin{array}{c}0.85 \\
6\end{array}$ & $\begin{array}{c}0.04 \\
2\end{array}$ & $0.773-0.939$ & 76.5 & 72.5 & $<0.0001^{* * * *}$ \\
\hline $\operatorname{miR}-146 a-5 p$ & $\begin{array}{c}0.79 \\
9\end{array}$ & $\begin{array}{c}0.05 \\
2\end{array}$ & $0.697-0.900$ & 64.7 & 62.5 & $<0.0001^{* * * *}$ \\
\hline miR-433-3p & $\begin{array}{c}0.83 \\
8\end{array}$ & $\begin{array}{c}0.04 \\
5\end{array}$ & $0.750-0.925$ & 70.6 & 67.5 & $<0.0001^{* * * *}$ \\
\hline$m i R-9-3 p+m i R-9-5 p$ & $\begin{array}{c}0.88 \\
9\end{array}$ & $\begin{array}{c}0.03 \\
6\end{array}$ & $0.818-0.960$ & 82.5 & 76.5 & $<0.0001^{* * * *}$ \\
\hline $\operatorname{miR}-9-3 p+m i R-146 a-5 p$ & $\begin{array}{c}0.90 \\
3\end{array}$ & $\begin{array}{c}0.03 \\
4\end{array}$ & $0.836-0.969$ & 80 & 76.5 & $<0.0001^{* * * *}$ \\
\hline$m i R-9-3 p+m i R-433-3 p$ & $\begin{array}{c}0.86 \\
5\end{array}$ & 0.04 & $0.787-0.944$ & 77.5 & 73.5 & $<0.0001^{* * * *}$ \\
\hline $\operatorname{miR}-9-5 p+\operatorname{miR}-146 a-5 p$ & $\begin{array}{c}0.90 \\
1\end{array}$ & $\begin{array}{c}0.03 \\
4\end{array}$ & $0.834-0.967$ & 80 & 79.4 & $<0.0001^{* * * *}$ \\
\hline$m i R-9-5 p+m i R-433-3 p$ & $\begin{array}{c}0.89 \\
8\end{array}$ & $\begin{array}{c}0.03 \\
4\end{array}$ & $0.830-0.965$ & 77.5 & 76.5 & $<0.0001^{* * * *}$ \\
\hline miR-146a-5p + miR-433-3p & $\begin{array}{c}0.88 \\
5\end{array}$ & $\begin{array}{c}0.03 \\
7\end{array}$ & $0.813-0.956$ & 77.5 & 73.5 & $<0.0001^{* * * *}$ \\
\hline$m i R-9-3 p+m i R-9-5 p+m i R-146 a-5 p$ & 0.93 & $\begin{array}{c}0.02 \\
7\end{array}$ & $0.877-0.983$ & 82.5 & 73.5 & $<0.0001^{* * * *}$ \\
\hline$m i R-9-3 p+m i R-9-5 p+m i R-433-3 p$ & $\begin{array}{c}0.91 \\
5\end{array}$ & 0.03 & $0.856-0.975$ & 80 & 79.4 & $<0.0001^{* * * *}$ \\
\hline$m i R-9-3 p+m i R-146 a-5 p+m i R-433-3 p$ & $\begin{array}{c}0.91 \\
8\end{array}$ & $\begin{array}{c}0.03 \\
1\end{array}$ & $0.858-0.979$ & 82.5 & 82.4 & $<0.0001^{* * * *}$ \\
\hline
\end{tabular}


miR-9-5p + miR-146a-5p + miR-433-3p

\begin{tabular}{cccccc}
0.92 & 0.02 & $0.871-0.980$ & 85 & 76.5 & $<0.0001^{* * * *}$ \\
6 & 8 & & & & \\
0.93 & 0.02 & $0.887-0.987$ & 82.5 & 76.5 & $<0.0001^{* * * *}$ \\
7 & 5 & & & & \\
\hline
\end{tabular}

$m i R-9-3 p+m i R-9-5 p+m i R-146 a-5 p+m i R-433-3 p$ 
Table 6(on next page)

Pairwise comparison of ROC curves. 
Pairwise comparison of ROC curves.

\begin{tabular}{|c|c|c|c|c|}
\hline Compared areas & $\begin{array}{c}\mathrm{DB} \\
\mathrm{A}\end{array}$ & SE & $95 \% \mathrm{Cl}$ & $\begin{array}{c}\mathrm{P} \\
\text { value }\end{array}$ \\
\hline miR-9-5p versus miR-9-5p + miR-9-3p & $\begin{array}{c}0.03 \\
4\end{array}$ & $\begin{array}{c}0.02 \\
7\end{array}$ & $\begin{array}{c}- \\
0.019 \sim 0.08 \\
8\end{array}$ & 0.212 \\
\hline miR-9-5p versus miR-9-5p + miR-146a-5p & $\begin{array}{c}0.04 \\
6\end{array}$ & $\begin{array}{c}0.03 \\
4\end{array}$ & $\begin{array}{c}- \\
0.021 \sim 0.11 \\
3\end{array}$ & 0.176 \\
\hline miR-9-5p versus miR-9-5p + miR-433-3p & $\begin{array}{c}0.04 \\
3\end{array}$ & $\begin{array}{c}0.02 \\
8\end{array}$ & $\begin{array}{c}- \\
0.012 \sim 0.09 \\
8\end{array}$ & 0.127 \\
\hline miR-9-5p + miR-9-3p versus miR-9-5p + miR-9-3p + miR-146a-5p & $\begin{array}{c}0.04 \\
1\end{array}$ & $\begin{array}{c}0.02 \\
5\end{array}$ & $\begin{array}{c}- \\
0.007 \sim 0.08 \\
9\end{array}$ & 0.094 \\
\hline miR-9-5p + miR-9-3p versus miR-9-5p + miR-9-3p + miR-433-3p & $\begin{array}{c}0.02 \\
6\end{array}$ & $\begin{array}{c}0.01 \\
8\end{array}$ & $\begin{array}{c}- \\
0.009 \sim 0.06 \\
2\end{array}$ & 0.143 \\
\hline miR-9-5p + miR-146a-5p versus miR-9-5p+miR-146a-5p + miR-9-3p & $\begin{array}{c}0.02 \\
9\end{array}$ & $\begin{array}{c}0.01 \\
9\end{array}$ & $\begin{array}{c}- \\
0.009 \sim 0.06 \\
8\end{array}$ & 0.131 \\
\hline miR-9-5p + miR-146a-5p versus miR-9-5p + miR-146a-5p + miR-433-3p & $\begin{array}{c}0.01 \\
5\end{array}$ & $\begin{array}{c}0.03 \\
3\end{array}$ & $\begin{array}{c}- \\
0.050 \sim 0.07 \\
9\end{array}$ & 0.654 \\
\hline$m i R-9-5 p+m i R-433-3 p$ versus miR-9-5p + miR-433-3p+miR-9-3p & $\begin{array}{c}0.01 \\
8\end{array}$ & $\begin{array}{c}0.01 \\
8\end{array}$ & $\begin{array}{c}- \\
0.017 \sim 0.05 \\
2\end{array}$ & 0.314 \\
\hline$m i R-9-5 p+m i R-433-3 p$ versus miR-9-5p+miR-433-3p+miR-146a-5p & $\begin{array}{c}0.02 \\
8\end{array}$ & $\begin{array}{c}0.02 \\
2\end{array}$ & $\begin{array}{c}- \\
0.016 \sim 0.07\end{array}$ & 0.21 \\
\hline
\end{tabular}


$m i R-9-5 p+m i R-9-3 p+m i R-146 a-5 p$ versus miR-9-5p + miR-9-3p+miR-146a-5p + miR-433-3p

$m i R-9-5 p+m i R-9-3 p+m i R-433-3 p$ versus miR-9-5p + miR-9-3p + miR-433-3p + miR-146a-5p

$m i R-9-5 p+m i R-146 a-5 p+m i R-433-3 p$ versus miR-9-5p + miR-146a-5p + miR-433-3p + miR-9-3p

\begin{tabular}{cccc}
0.00 & 0.01 & $0.013 \sim 0.02$ & 0.514 \\
7 & \multicolumn{3}{c}{6} \\
& & - & \\
0.02 & 0.02 & $0.018 \sim 0.06$ & 0.288 \\
1 & & 1 & \\
& & - & \\
0.01 & 0.01 & $0.013 \sim 0.03$ & 0.378 \\
1 & 3 & 6 & \\
0.07 & 0.03 & $0.009 \sim 0.14$ & 0.026 \\
5 & 4 & 2 & $*$ \\
0.06 & 0.03 & $0.001 \sim 0.12$ & 0.045 \\
1 & & 0 & $*$ \\
0.07 & 0.03 & $0.005 \sim 0.13$ & 0.034 \\
1 & 4 & 7 & $*$ \\
0.08 & 0.03 & $0.015 \sim 0.14$ & 0.016 \\
2 & 4 & 9 & $*$ \\
0.04 & 0.02 & - & \\
8 & 6 & $0.003 \sim 0.09$ & 0.066 \\
& & 9 & \\
0.03 & 0.02 & - & \\
6 & 1 & $0.004 \sim 0.07$ & 0.081 \\
& & 7 & \\
0.03 & 0.02 & $-0.011 \sim 0.08$ & 0.124 \\
9 & 5 & 9 & \\
\hline & & &
\end{tabular}




\section{Table 7 (on next page)}

Four selected miRNAs and their combinations in three public database for prognostic analysis. 
Four selected miRNAs and their combinations in three public databases for prognostic analysis.

\begin{tabular}{lccc}
\hline \multicolumn{1}{c}{ miRNA and Combination } & Oncoln & OncomiR & Kaplan-Meier Plotter \\
& c & & \\
\cline { 2 - 4 } & P-value & P-value & P-value \\
\hline miR-9-3p & $0.0084^{* *}$ & 0.0664 & $0.0047^{* *}$ \\
miR-9-5p & $0.0018^{\star *}$ & $0.0044^{* *}$ & $0.0047^{\star *}$ \\
miR-146a-5p & 0.1460 & $0.0460^{*}$ & $0.0230^{*}$ \\
miR-433-3p & $0.0311^{*}$ & 0.0565 & $0.0017^{* *}$ \\
miR-9-3p + miR-9-5p & None & $0.0034^{* *}$ & $0.0047^{* *}$ \\
miR-9-3p + miR-146a-5p & None & 0.6535 & $0.0017^{* *}$ \\
miR-9-3p + miR-433-3p & None & 0.2405 & $0.0047^{* *}$ \\
miR-9-5p + miR-146a-5p & None & 0.3989 & $0.0017^{* *}$ \\
miR-9-5p + miR-433-3p & None & $0.0143^{*}$ & $0.0047^{* *}$ \\
miR-146a-5p + miR-433-3p & None & 0.1278 & $0.0230^{*}$ \\
miR-9-3p + miR-9-5p + miR-146a-5p & None & 0.6655 & $0.0017^{* *}$ \\
miR-9-3p + miR-9-5p + miR-433-3p & None & $0.0081^{* *}$ & $0.0047^{* *}$ \\
miR-9-3p + miR-146a-5p + miR-433-3p & None & 0.5808 & $0.0020^{* *}$ \\
miR-9-5p + miR-146a-5p + miR-433-3p & None & 0.5782 & $0.0020^{* *}$ \\
miR-9-3p + miR-9-5p + miR-146a-5p + miR-433-3p & None & 0.4551 & $0.0020^{* *}$ \\
\hline
\end{tabular}

